\title{
IL-21 signaling promotes early dissemination of KSHV infection in B lymphocytes
}

Nedaa Alomari ${ }^{1}$, Farizeh Aalam ${ }^{1}$, Romina Nabiee ${ }^{1}$, Jesus Ramirez Castano ${ }^{1}$ and Jennifer Totonchy

${ }^{1}$ Biomedical and Pharmaceutical Sciences, Chapman University, Irvine, CA USA

†Corresponding Author

Jennifer Totonchy

Chapman University School of Pharmacy

9410 Jeronimo Road

Irvine, CA

Phone: 714-516-5438

Email: totonchy@chapman.edu

Short Title: IL-21 and KSHV infection of B cells 


\section{Abstract}

2 Kaposi's sarcoma-associated herpesvirus (KSHV) extensively manipulates the host immune

3 system and the cytokine milieu, and cytokines are known to influence the progression of KSHV-

4 associated diseases. However, the precise role of cytokines in the early stages of KSHV infection

5 remains undefined. Here, using our unique model of KSHV infection in tonsil lymphocytes, we

6 investigate the influence of host cytokines on the establishment of KSHV infection in B cells.

7 Our data demonstrate that KSHV manipulates the host cytokine microenvironment during early

8 infection and susceptibility generally associated with downregulation of multiple cytokines.

9 However, we show that IL-21 signaling promotes KSHV infection by promoting both plasma

10 cell numbers and increasing KSHV infection in plasma cells. Our data reveal that IL-21

11 producing T cells, particularly Th17/Tc17 and central memory CD8+ T cells may represent

12 immunological factors that modulate host-level susceptibility to KSHV infection. These results

13 suggest that IL-21 plays a significant role in the early stages of KSHV infection in the human

14 immune system and may represent a novel mechanism to be further explored in the context of

15 preventing KSHV transmission.

\section{Author Summary}

17 Very little is known about how KSHV is transmitted and how it initially establishes infection in a

18 new human host and this lack of information limits our ability to prevent KSHV-associated cancers

19 by limiting its person-to-person transmission. Saliva is thought to be the primary route of person-

20 to-person transmission for KSHV, making the tonsil a likely first site for KSHV replication in a

21 new human host. In particular, the tonsil is likely to be the first place KSHV is able to enter B

22 cells, which are thought to be a major site of persistent infection. Our previous work identified 
23 plasma cells as a highly targeted cell type in early KSHV infection in cultured cells from human

24 tonsil. In this study, we show that the human cytokine IL-21 promotes both overall KSHV infection

25 and the establishment of infection in plasma cells. We also investigate the immunological

26 mechanisms underlying this effect. Our results demonstrate that IL-21 and IL-21-producing cells

27 are a novel factor that influences the initial establishment of KSHV infection in humans.

Introduction

29 Kaposi's Sarcoma Herpesvirus (KSHV) is a lymphotropic gamma-herpesvirus, originally

30 discovered as the causative agent of Kaposi Sarcoma (KS) [1]. KS is a highly proliferative tumor

31 derived from lymphatic endothelial cells [2]. KSHV is also associated with the B cell

32 lymphoproliferative diseases, Primary Effusion Lymphoma (PEL) and Multicentric Castleman's

33 Disease (MCD) [3, 4], as well as the inflammatory disorder KSHV inflammatory cytokine

34 syndrome (KICS) [5]. KSHV is linked to $1 \%$ of all human tumors, and the World Health

35 Organization (WHO) has classified it as class I carcinogen [6, 7]. KSHV infection is asymptomatic

36 in most healthy individuals, and KSHV-associated malignancies arise primarily in

37 immunocompromised patients. Indeed, KS remains one of the most common cancers in people

38 living with HIV/AIDS [8].

39 The geographical distribution of KSHV is not ubiquitous. KSHV infection is endemic in sub-

40 Saharan Africa and in the Mediterranean basin. KSHV prevalence is also high in subpopulations

41 in other parts of the world such as men who have sex with men (MSM). Saliva is the only secretion

42 where KSHV DNA is commonly detected [9], and, based on this, person-to-person transmission

43 of KSHV is thought to occur via saliva. The oral lymphoid tissues are rich in KSHV target cell

44 types including lymphatic endothelial cells and B cells, and are therefore a likely site for the initial 
45 establishment of KSHV infection in a new human host. However, the exact mechanisms for KSHV

46 transmission and how environmental, behavioral and host factors influence transmission and early

47 infection events remain to be established. This gap in our understanding dramatically affects our

48 ability to find efficient strategies to decrease the transmission or influence host-level susceptibility

49 to KSHV infection. One of the main factors that has been linked to KSHV-associated

50 lymphoproliferations is cytokine dysregulation [10]. However, their contribution to the early

51 stages of KSHV infection and whether the cytokine milieu in the oral cavity contributes to host-

52 level susceptibility to KSHV infection is unclear. KSHV-infected cells in KSHV-associated

53 lymphoproliferative diseases generally display plasma cell or plasmablast features [11], and our

54 recent work showed that KSHV targets plasma cells in early infection in tonsil B lymphocytes

55 [12]. Whether the KSHV-infected plasma cells we observe arise from direct infection or

56 differentiation from a precursor B cell subset remains to be established. In either case, it is likely

57 that cytokines play a role in supporting the survival and/or differentiation of KSHV-infected 58 plasma cells.

59 IL-21 is a pleiotropic cytokine that has diverse effects on B cell, T cell, macrophage, monocyte,

60 and dendritic cell biology. It is produced mainly by natural killer T (NKT) cells and CD4+ T cells,

61 including follicular helper (TFH) cells [13]. The IL-21 receptor is expressed by several immune

62 cells, including $\mathrm{B}$ and $\mathrm{T}$ cells and is comprised of a unique IL-21R subunit and the common

63 cytokine receptor $\gamma$ chain (CD132), which is also part of the receptor for IL-2, IL-4, IL-7, IL-9,

64 and IL-15 [14]. IL-21 plays a critical role in B cell activation and expansion [15], and plays a

65 critical role in B cell differentiation to immunoglobulin (Ig)-secreting plasma cells. The regulation

66 of maturation of B cells into plasma cell is driven by the several transcription factors including 
67 Blimp1 and Bcl6 [16], which can both be induced by IL-21 signaling, indicating that IL-21 is an

68 important regulator of plasma cell differentiation $[17,18]$.

69 There are few studies to date examining the contribution of IL-21 to KSHV infection and KSHV-

70 associated disease. However, IL-21 is detected in interfollicular areas in MCD patients [19].

71 Moreover, IL-21 induces differentiation of B-lymphoblastoid cell lines (BCLs) into late

72 plasmablasts/early plasma cells and regulates the expression of many latent proteins in

$73 \mathrm{EBV}^{+}$Burkitt lymphoma cell lines [20, 21]. Importantly, IL-21 signaling is required for the

74 establishment of MHV-68 infection and the generation for MHV-68 infected long-lived plasma

75 cells in mice [22].

76 In this study, we use our well-established tonsil lymphocyte infection model to explore whether

77 KSHV alters cytokine secretion early in infection and whether cytokine levels have an effect on

78 the establishment of KSHV infection. We identify IL-21 as a factor that specifically influences

79 KSHV infection in plasma cells, which we previously characterized as a highly targeted cell type

80 in early infection [12]. We demonstrate that IL-21 signaling increases plasma cell levels in our ex

81 vivo model system and that this effect promotes overall KSHV infection. We explore the

82 mechanisms of IL-21 signaling by establishing which B cell types are responding IL-21 and what

83 T cell subsets are producing IL-21 in our model.

84 We determine that expression of IL-21 receptor (IL-21R) on naïve and classical memory T cells

85 is correlated with increased KSHV infection in IL-21 treated cultures, and we demonstrate that

86 both KSHV infection and IL-21 treatment modulates IL-21R expression on B cells. We examine

87 IL-21 secretion by $\mathrm{T}$ cell subsets in our tonsil lymphocyte cultures and demonstrate that the

88 magnitude and distribution of IL-21 secretion varies considerably based on tonsil donor, but is not

89 significantly modulated by KSHV infection. Interestingly, RoR $\gamma \mathrm{T}+\mathrm{T}$ cells (both CD4+ and CD8+) 
90 contributed substantially to IL-21 secretion and were positively correlated with KSHV infection

91 of plasma cells at 3 dpi. Moreover, IL-21 secretion by CD8+ central memory T cells was positively

92 correlated with both overall KSHV infection and plasma cell targeting.

93 These results identify IL-21 signaling as a factor that influences the establishment of KSHV

94 infection in B lymphocytes and, together with our previous work, underscores the importance of

95 plasma cell biology in the initial establishment of KSHV infection in the oral lymphoid tissues.

96 Based upon this work, we conclude that specific IL-21 secreting $\mathrm{T}$ cell subsets represent an

97 important susceptibility factor for KSHV transmission.

\section{Results}

\section{Host cytokines influence the establishment of KSHV infection B lymphocytes}

100 Despite the critical interplay between KSHV and host cytokine signaling, little is known about

101 whether cytokines influence host susceptibility to KSHV infection. In fact, the roles of

102 proinflammatory cytokines during KSHV infection have been studied mostly in naturally-infected

103 human B cell lines derived from PEL [23, 24]. In order to examine whether cytokines alter the

104 early stages of KSHV infection in the tonsil, we quantitated the levels of 13 cytokines in the

105 supernatants of Mock and KSHV-infected tonsil lymphocyte cultures at 3 days post-infection

106 using a bead-based multiplex immunoassay. This dataset includes 33 independent infections using

10724 unique tonsil specimens. IL-6, IFNg, TNFa and IL-22 were the most prevalent cytokines in our

108 cultures based on the median values overall (Fig 1A, panel order). IL-6 was the only cytokine

109 significantly induced in KSHV-infected cultures compared to Mock cultures (Fig 1A) and the

110 magnitude of IL-6 induction by KSHV infection is far greater than the effect of infection on any 
111 other cytokine (Fig 1B). This result is consistent with our previous results in cultures containing

112 only naïve B lymphocytes [25]. Our data also reveals statistically significant reductions in IL-5

113 and IL-4 concentrations in KSHV-infected cultures compared to Mock cultures (Fig 1A), but these

114 changes are very small compared to those seen with IL-6 (Fig 1B). Interestingly, IFNg

115 concentrations were highly affected by KSHV infection, but there were sample-specific

116 differences in whether this effect was positive or negative (Fig 1B).

117 In order to determine whether cytokines affect the establishment of KSHV infection, we examined

118 whether the concentration of cytokines in the supernatants of KSHV-infected cultures is correlated

119 with the level of infection in B lymphocytes (based on GFP reporter expression) in the same culture

120 by flow cytometry analysis (Fig 1C \& D). On a per-sample level, many individual cytokines

121 (notably IL-6 and IFNg) were induced or repressed independent of susceptibility. However, there

122 is a cluster of highly susceptible samples (ND19, ND40, ND41, ND32, ND4) in which multiple

123 cytokines are repressed in the KSHV-infected cultures (Fig 1C). Pairwise comparisons between

124 overall GFP level in B lymphocytes and the level of each cytokine in the KSHV-infected cultures

125 revealed universally negative correlations between overall KSHV infection and cytokine levels

126 with lower cytokine levels observed in more susceptible samples. These negative correlations were

127 statistically significant for IL-2, IL-9, IL-10, TNFa, IL-4 and IL-22 (Fig 1D). Since plasma cells

128 were identified as a highly targeted cell type in our previous study [12], we examined the

129 correlations between cytokine levels in KSHV-infected cultures and infection in the CD138+

130 plasma cell subset. This analysis revealed negative correlations similar to those seen with overall

131 infection with IL-13, IL-9, IFNg, TNFa and IL-22 levels showing statistically significant negative

132 correlations with plasma cell infection. However, in this analysis IL-21 levels showed a significant

133 positive correlation with plasma cell infection (Fig 1E). Taken together, these data 
134 demonstrate that (1) KSHV infection influences the production of multiple cytokines in our ex vivo

135 infection model, (2) lower cytokine levels and/or repression of cytokines during infection are

136 generally associated with higher susceptibility to KSHV infection, (3) several individual cytokines

137 show significant negative associations with susceptibility to KSHV infection and (4) IL-21 is

138 positively correlated with KSHV infection of plasma cells. Overall, these data suggest that distinct

139 inflammatory responses in each tonsil specimen contribute to variable susceptibility to KSHV

140 infection.

141 IL-21 supplementation increases KSHV infection in tonsil B lymphocytes

142 Because IL-21 production was positively correlated with plasma cell infection in our initial dataset

143 (Fig 1E), we wanted to examine the impact of manipulating IL-21 levels on the establishment of

144 KSHV infection. To do this, we performed Mock infection or KSHV infection in 12 unique tonsil

145 samples and supplemented the resulting cultures with varying concentrations of recombinant IL-

146 21. At 3 dpi, we analyzed these cultures for GFP+ B lymphocytes by flow cytometry to assess the

147 magnitude of KSHV infection (Fig 2A \& B). Although the specimens included in this data set had

148 high variability in their baseline susceptibility, we can see increased infection in response to IL-

14921 treatment, and the effect seems to be particularly strong in the more susceptible samples (Fig

$1502 \mathrm{~A})$. Normalization of the data to each specimen's untreated control reveals that at 10/12 samples

151 show increased infection upon treatment with $100 \mathrm{pg} / \mathrm{ml}$ of IL-21. Importantly, most of these

152 concentrations were higher than what was observed in our initial dataset quantitating native

153 cytokine secretion in our culture system (Fig 1A), which may explain why we didn't observe an

154 association of IL-21 secretion with overall infection in that data (Fig 1D). We then repeated these

155 supplementation experiments with only the $100 \mathrm{pg} / \mathrm{ml}$ dose of recombinant IL-21 in an additional 
15612 tonsil specimens and examined both overall infection and subset-specific responses in these

157 cultures at 3 dpi using our B cell immunophenotyping panel. Similar to the initial dataset, this

158 analysis shows increased infection in response to recombinant IL-21 in the majority of tonsils, and

159 the difference in GFP+ B lymphocytes was statistically significant in IL-21 treatment compared to

160 control $(\mathrm{p}=0.02, \mathrm{~F}=6.4)($ Fig $2 \mathrm{C})$.

161 IL-21 increases plasma cell frequency and susceptibility in primary human tonsil B

162 lymphocytes

163 In order to determine whether IL-21 signaling influences the overall distribution of B cell subsets

164 in both Mock and KSHV infected cultures, we performed B cell immunophenotyping. Analysis of

165 overall subset frequencies, with all subsets represented as a fraction of viable CD19+ B cells, at 3

166 dpi revealed that most subsets did not change with either KSHV infection or IL-21 treatment. We

167 did observe a statistically significant increase in CD20+ B cells and plasmablasts in the KSHV-

168 infected conditions independent of IL-21 treatment. Naïve B cells showed significant main effects

169 for both infection and treatment, with both KSHV infection and IL-21 treatment reducing naïve B

170 cells in the culture, but the interaction was not significant. Importantly, this analysis revealed a

171 highly significant increase in total plasma cell frequency associated with both IL-21 treatment and

172 KSHV infection with a significant interaction of the two variables. This effect seems to be

173 restricted to CD20+ plasma cells in the mock conditions and distributed between both CD20+ and

174 CD20- plasma cells in the KSHV-infected cultures. However, both CD20+ and CD20- plasma

175 cells showed statistically significant interactions between infection and treatment (Fig 2D, Table

176 1). Post hoc paired T tests revealed significant differences with IL-21 treatment on total plasma

177 cells $(\mathrm{p}=0.0002)$ and CD20+ plasma cells $(\mathrm{p}=0.002)$ for the KSHV-infected conditions only. The 
178 IL-21 effect on naïve B cell frequencies was significant for both Mock $(\mathrm{p}=0.01)$ and KSHV

179 infected conditions $(\mathrm{p}=0.003)$. Moreover, in IL-21 treated conditions there was a significant

180 difference between Mock and KSHV cultures for total plasma cells ( $p=0.0003)$, CD20+ plasma

181 cells $(\mathrm{p}=0.02)$ and naïve $\mathrm{B}$ cells $(\mathrm{p}=0.006)$. One interpretation of this data is that IL-21 can

182 independently drive plasma cell differentiation of B cells in our culture system resulting in

183 decreased naïve cells and increased plasmablast and plasma cells, and KSHV infection acts

184 synergistically with IL-21 to potentiate the same effect. We next wanted to determine whether IL-

18521 treatment altered the B cell subset-specific distribution of KSHV infection. For this analysis,

186 we quantitated the percent of each B cell subset that was GFP+ to determine the within-subsets

187 distribution of KSHV infection in control or IL-21 treated cultures (Fig 2E). In this analysis, we

188 observed a significant increase in plasma cell targeting with IL-21 treatment $(\mathrm{p}=0.02, \mathrm{~F}=6.6)$. We

189 next examined whether increased frequency of plasma cells or increased plasma cell targeting was

190 directly correlated with the observed increase in overall KSHV infection in the IL-21 treated

191 conditions (Fig 2F). These results reveal a significant linear correlation between total GFP and

192 plasma cell frequency $(\mathrm{r}=0.7, \mathrm{p}=0.007)$ and a weaker correlation between total GFP and the

193 frequency of GFP+ cells within the plasma cell subset $(r=0.56, p=0.04)$.

194 Taken together this data shows that IL-21 treatment promotes the establishment of KSHV infection

195 in human tonsil lymphocytes and that this increased infection is correlated with both increased

196 plasma cell frequencies and increased plasma cell infection. Thus, our results suggest that IL-21

197 signaling facilitates the establishment of KSHV infection by driving the proliferation, survival or

198 differentiation of plasma cells in our tonsil lymphocyte cultures. The observation that naïve B cell

199 frequencies are significantly decreased in KSHV-infected, IL-21-treated cultures (Fig 2D) 
200 supports the latter conclusion that increased plasma cell frequencies are a result of B cell

201 differentiation in response to IL-21 signaling.

202 Table 1: Statistically significant effects from two-way repeated measures ANOVA analysis

203 in Fig 2D

\begin{tabular}{|l|l|l|l|l|l|l|}
\hline Variable & Effect & DFn & DFd & F & p & ges \\
\hline GFP & Treatment & 1 & 13 & 6.425 & 0.025 & 0.081 \\
\hline GFP & Infection & 1 & 13 & 102.896 & $1.52 \mathrm{e}-07$ & 0.708 \\
\hline GFP & Tx:Inf & 1 & 13 & 7.635 & 0.016 & 0.084 \\
\hline Plasma cells & Treatment & 1 & 13 & 18.596 & 0.000844 & 0.163 \\
\hline Plasma cells & Infection & 1 & 13 & 18.575 & 0.000848 & 0.166 \\
\hline Plasma cells (PC) & Tx:Inf & 1 & 13 & 22.779 & 0.000364 & 0.09 \\
\hline Naive & Treatment & 1 & 13 & 13.643 & 0.003 & 0.099 \\
\hline Naive & Infection & 1 & 13 & 8.462 & 0.012 & 0.069 \\
\hline Plasmablast & Infection & 1 & 13 & 6.023 & 0.029 & 0.02 \\
\hline CD20- PC & Tx:Inf & 1 & 13 & 12.645 & 0.004 & 0.043 \\
\hline All & Infection & 1 & 13 & 6.234 & 0.027 & 0.016 \\
\hline CD20+ PC & Treatment & 1 & 13 & 4.858 & 0.046 & 0.028 \\
\hline & & & & & & \\
\hline
\end{tabular}


206 We next wanted to determine whether neutralization of the natively-secreted IL-21 in our tonsil

207 lymphocyte cultures would affect the establishment of KSHV infection. To do this we performed

208 infections with Mock or KSHV-infection in 11 unique tonsil specimens, included varying

209 concentrations of an IL-21 neutralizing antibody in the resulting cultures, and assessed the

210 magnitude and distribution of KSHV infection at 3 dpi by flow cytometry. These results revealed

211 decreased KSHV infection in the presence of IL-21 neutralizing antibodies (Fig 3A). One-way

212 repeated measures ANOVA revealed a significant effect of IL-21 neutralization on GFP in KSHV

213 infected cultures $(\mathrm{p}=0.00001, \mathrm{~F}=9.4)$ and post-hoc Dunnett test revealed significance at the

$214100 \mu \mathrm{g} / \mathrm{ml}$ dose $(\mathrm{p}=0.03)$. When each sample was normalized to its untreated control, we observed

215 that 9/11 samples had decreased infection in the presence of $100 \mu \mathrm{g} / \mathrm{ml} \mathrm{IL-21} \mathrm{neutralizing} \mathrm{antibody}$

216 and this increased to 10/11 samples at higher antibody doses (Fig 3B).

217 When we examined the overall frequency of B cell subsets at 3dpi in these experiments, we

218 observed statistically significant effects of IL-21 neutralization on a number of B cell subsets, but

219 there were no significant interaction effects with IL-21 neutralization and KSHV infection using

220 two-way repeated measures ANOVA (Fig 3C). Specifically, IL-21 neutralization increased IgG+

$221(\mathrm{p}=0.04, \mathrm{~F}=5.0)$, memory $(\mathrm{p}=0.03, \mathrm{~F}=3.4)$, and double negative $(\mathrm{p}=0.02, \mathrm{~F}=3.8) \mathrm{B}$ cells and

222 decreased $\operatorname{IgM}+(\mathrm{p}=0.0003, \mathrm{~F}=8.6)$ and transitional $(\mathrm{p}=0.01, \mathrm{~F}=4.1)$ subsets. Post-hoc paired $\mathrm{T}$ -

223 tests showed a statistically significant decrease of on both overall $(\mathrm{p}=0.02)$ and CD20+ $(\mathrm{p}=0.005)$

224 plasma cell frequencies at the $200 \mu \mathrm{g} / \mathrm{ml}$ dose in the mock infected cultures only. The observation

225 that the effect of IL-21 neutralization on plasma cell frequencies is restricted to mock infected

226 cultures is interesting in the context of our IL-21 supplementation data where we observed

227 significant main effects of both IL-21 and KSHV infection on plasma cell frequencies as well as a

228 significant interaction between the two factors (Fig 2D \& Table 1). The two data sets taken together 
support several interesting conclusions: (1) IL-21 affects plasma cell frequencies independent of

230 KSHV infection; evidenced by opposite significant effects of IL-21 supplementation and

231 neutralization in mock cultures, (2) KSHV infection affects plasma cell frequencies independent

232 of IL-21 signaling; evidenced by significant main effect of infection in supplemented cultures and

233 a lack of inhibition in KSHV-infected neutralized cultures, and (3) IL-21 and KSHV can

234 synergistically affect plasma cell frequencies; evidenced by the significant interaction effect and

235 the significant increase in plasma cell frequencies in $\mathrm{KSHV}$-infected cultures that are

236 supplemented with IL-21.

237 We next wanted to determine whether neutralization of IL-21 influences the frequency of KSHV

238 infection within B cell subsets. One-way repeated measures ANOVA revealed a significant effect

239 of neutralization on infection of transitional $\mathrm{B}$ cells $(\mathrm{p}=0.01, \mathrm{~F}=4.0)$ but paired $\mathrm{T}$ tests were not

240 significant for any individual dose. When we plotted this data normalized to the per-sample, per-

241 subset infection rate of the untreated control cultures, we observed that neutralization was

242 associated with lower levels of infection in transitional, plasmablast, and CD20+ PC populations

243 (Fig 3D). We hypothesized that if IL-21 signaling is affecting overall infection by contributing

244 substantially to differentiation of KSHV-infected cells, we might observe a correlation between

245 overall infection and the contribution of specific subsets to infection within neutralized cultures

246 compared to control cultures. If this hypothesis is correct, subsets whose differentiation is

247 important to the establishment of infection would accumulate with IL-21 neutralization and this

248 accumulation would correlate with decreased overall levels of infection in the same cultures. To

249 examine this, we calculated the change in IL-21 neutralized cultures compared to control cultures

250 (neutralized-control) for both overall infection and the contribution of each subset to infection

251 (between subsets frequency of GFP), and performed correlation analysis using Pearson's method. 
252 This analysis reveals that decreased overall infection with IL-21 neutralization was significantly

253 correlated with a decreased contribution of plasma cells $(r=0.6, p=0.0002), C D 20+$ plasma cells

$254(\mathrm{r}=0.6, \mathrm{p}=0.0002)$, and transitional $\mathrm{B}$ cells $(\mathrm{r}=0.5, \mathrm{p}=0.003)$ and an increased proportion of infected

255 germinal center cells $(\mathrm{r}=-0.4, \mathrm{p}=0.02)$ (Fig 3E). Interestingly, these correlations were driven more

256 by sample-specific differences (indicated by point color) compared to dose response of

257 neutralization (indicated by point shape). This data could indicate that IL-21 signaling increases

258 the overall establishment of KSHV infection in tonsil lymphocytes by driving differentiation of

259 KSHV-infected germinal center cells into transitional and CD20+ plasma cells. Our previous

260 studies demonstrated that plasma cells display a mixture of lytic and latent KSHV infection [12].

261 Therefore, we wanted to determine whether the increase in overall KSHV infection with IL-21

262 treatment and decrease in infection with IL-21 neutralization is due to IL-21-mediated alterations

263 in KSHV lytic reactivation. To examine this, we performed RT-PCR for LANA (latent) and K8.1

264 (lytic) on total RNA from untreated, IL-21 supplemented or IL-21 neutralizing antibody treated,

265 KSHV-infected cultures from 8 unique tonsil specimens. GAPDH was used as a housekeeping

266 gene and normalizing factor for the viral gene expression data. This data is consistent with our

267 previous data showing a mix of lytic and latent transcripts in infected lymphocytes [12]. These

268 data reveal no significant influence of either supplementation or neutralization on lytic gene

269 expression (Fig 3F). In the majority of samples K8.1 expression remained unchanged or changes

270 were also reflected in LANA transcripts (Fig 3G).

271 IL21 receptor distribution in primary human tonsil B lymphocytes affects the magnitude

272 and distribution of KSHV infection 
273 Our data presented thus far demonstrates that IL-21 signaling has a positive effect on the overall

274 establishment of KSHV infection (Fig 2C and 3B) and this increase in overall infection is related

275 to both the absolute number of plasma cells (Fig 2D\&F and 3C), and the establishment of infection

276 in plasma cells (Fig 2E\&F and 3E). Moreover, our data suggests that the increase in plasma cell

277 numbers and targeting may be due to differentiation of new plasma cells via a process that requires

278 IL-21 signaling to germinal center B cells (Fig 3E). To further address the early stages of the IL-

27921 response during infection, we examined expression of the IL21 receptor in primary human

280 tonsil B lymphocytes at baseline (day 0) in each tonsil specimen. We observed that IL-21 receptor

281 expression is rare on B cells in tonsil at less than $3 \%$ of total viable B cells in most samples (Fig

282 4A). The distribution of IL-21 receptor positive cells among B cell sub-populations is broad, but

283 IL-21 receptor-expressing B cells are most likely to have an MZ-like or plasmablast

284 immunophenotype (Fig 4B) and on a per-sample basis, either plasmablast or MZ-like subsets

285 dominated the IL-21R positive cells in most tonsil samples (Fig 4C)

286 In order to determine whether IL-21 receptor expression at baseline influenced the establishment

287 of KSHV infection, we aggregated the untreated conditions from both the supplementation and the

288 neutralization experiments and examined correlations between baseline IL21R distribution and

289 KSHV infection based on overall GFP. This analysis revealed that the proportion of plasmablasts

290 within IL21R+ B cells is significantly correlated with overall susceptibility to KSHV infection

291 ( $\mathrm{r}=0.81, \mathrm{p}=0.0007)$ (Fig 4D). In experiments where we supplemented cultures with IL-21, the

292 positive effect of IL-21 treatment on overall KSHV infection at 3 dpi (Fig 2C) is correlated with

293 the baseline frequency of IL-21 receptor expression on naïve B cells $(r=0.82, p=0.01)$ and classical

294 memory B cells ( $\mathrm{r}=0.7, \mathrm{p}=0.05)$ (Fig 4E). The significant correlation between the IL-21 response

295 and baseline IL-21 receptor expression is particularly interesting and consistent with our 
296 hypothesis that differentiation of naïve B cells into plasma cells contributes to the increase in

297 overall infection we see with IL-21 treatment (Fig 2D).

298 When we correlated baseline IL21R expression with the positive effect of IL-21 treatment on total 299 plasma cell numbers in KSHV-infected cultures (Fig 2D), no significant positive correlations were

300 found (Fig 4F). This lack of correlation suggests that the plasma cell response to IL-21 at 3 dpi in

301 KSHV infected cultures may be a product of IL-21 receptor up-regulation in response to infection

302 instead of intrinsic baseline levels of IL-21 on plasma cells or plasma cell precursors in our tonsil

303 lymphocyte cultures. Indeed, modulation of IL-21 receptor expression by KSHV infection could

304 be one mechanism for the synergistic promotion of plasma cell numbers we observe with both IL-

30521 treatment and infection (Fig 2D).

306 In order to examine this hypothesis, we analyzed IL-21R expression on B cell subsets at 3 dpi in

307 our culture system with or without IL-21 supplementation to determine whether KSHV and/or IL-

30821 can modulate the response to IL-21 during infection. These results reveal that the majority of

309 effects on IL-21R at 3 dpi are present in both Mock and KSHV-infected cultures, indicating they

310 are a product of the culture system and not driven by KSHV. However, the proportion of

311 plasmablasts within IL21R+ cells was significantly different comparing Mock to KSHV-infected

312 cultures without IL-21 treatment $(\mathrm{p}=0.03)$ and this difference was increased with the combination

313 of KSHV infection and IL-21 treatment (Fig 4G). This result may indicate that infection is

314 affecting IL21R expression on plasmablasts or that KSHV is infecting a precursor B cell and

315 driving differentiation of these cells into IL21R+ plasmablasts. Taken together with the fact that

316 IL-21R expression on plasmablasts at day 0 is highly correlated with overall infection (Fig 4D),

317 these data strongly suggest that plasmablasts play a role in the response of KSHV infection to IL- 
21 treatment. Although not statistically significant, a similar trend can be observed for IL21R+

319 plasma cells while the converse trend is seen for IL21R positive germinal center cells (Fig 4G),

320 these observations are consistent with the data from our IL21 neutralization studies that suggests

321 germinal center cells may be differentiating into plasma cells in response to IL-21 signaling (Fig

$3223 \mathrm{E})$, potentially via a plasmablast intermediate phenotype.

\section{Characterization of T cell subsets producing IL-21 in primary human tonsil B lymphocytes}

324 We next wanted to determine the source of native IL-21 secretion in our culture system, and 325 determine whether the production of IL-21 is affected by KSHV infection. To accomplish this, we 326 utilized an additional immunophenotyping panel for T cell subsets (Table 2 and Supplemental Fig 327 1) and performed intracellular cytokine staining (ICCS) to identify IL-21 producing T cells at 3 328 dpi in Mock and KHSV-infected cultures from 14 unique tonsil samples. This data reveals that IL-

32921 secretion in T cells is highly variable between tonsil lymphocyte cultures, but is not significantly

330 affected by KSHV infection (Fig 5A). More of the IL-21+ cells were CD4+ T cells vs. CD8+ T 331 cells and this distribution was also not affected by KSHV infection (Fig 5B). When we examined 332 the subset-level distribution of IL-21 secretion within T cells, we observed that within CD4+ cells 333 CD45RO+, central memory, TFH and RoR $\gamma \mathrm{T}+$ cells were contributing most to IL-21 secretion. 334 Among CD8+ T cells, CD45RA+, stem cell memory, central memory and RoR $\gamma \mathrm{T}+$ cells were the 335 highest contributors to IL-21 secretion (Fig 5C). When we examined whether KSHV infection 336 altered the contribution of T cell subsets to IL-21 secretion in these cultures, we found that the 337 contribution of CD4+ CD45RA+, CD4+ RoR $\gamma \mathrm{T}+$ and CD8+ central memory subsets was 338 significantly decreased in KSHV-infected cultures vs. Mock cultures (Fig 5D). However, KSHV 339 infection did not significantly change the overall levels and subset distribution of $\mathrm{T}$ cells within 
340 these cultures, indicating these are biological changes within T cell subsets and not due to changes

341 in the T cell population during infection (Supplemental Fig 2).

342

Table 2: Lineage definitions for lymphocyte subsets used in the study

\section{B Lymphocytes}

\begin{tabular}{|c|c|}
\hline Subset & Molecular Markers \\
\hline Plasma & $\mathrm{CD}_{19}{ }^{+}, \mathrm{CD} 20^{+/-}, \mathrm{CD} 138^{+(\text {Mid to High })}, \mathrm{CD}^{-} 8^{-}$ \\
\hline Transitional & $\mathrm{CD} 19^{+}, \mathrm{CD} 138^{-}, \mathrm{CD} 38^{\mathrm{Mid}}, \mathrm{IgD}^{+}$(Mid to High) \\
\hline Plasmablast & $\mathrm{CD}_{19}+, \mathrm{CD} 138^{-}, \mathrm{CD} 38^{\mathrm{High}}, \mathrm{IgD}^{+/-(\text {mostly }-)}$ \\
\hline Germinal Center & $\mathrm{CD} 19^{+}, \mathrm{CD} 138^{-}, \mathrm{CD} 38^{\mathrm{Mid}}, \mathrm{IgD}^{-}$ \\
\hline Naïve & $\mathrm{CD} 9^{+}, \mathrm{CD} 138^{-}, \mathrm{CD} 38^{\mathrm{Low}}, \mathrm{CD} 27^{-}, \mathrm{IgD}^{+ \text {(Mid to High) }}$ \\
\hline $\begin{array}{l}\text { Marginal Zone Like } \\
\text { (MZ-Like) }\end{array}$ & $\mathrm{CD} 19^{+}, \mathrm{CD} 138^{-}, \mathrm{CD} 38^{\text {Low }}, \mathrm{CD} 27^{+ \text {(Mid to High) }}, \mathrm{IgD}^{+(\text {Mid to High) }}$ \\
\hline Memory & $\mathrm{CD} 9^{+}, \mathrm{CD} 138^{-}, \mathrm{CD} 38^{\text {Low }}, \mathrm{CD} 27^{+ \text {(Mid to High) }}, \mathrm{IgD}^{-}$ \\
\hline Double Negative & $\mathrm{CD}^{+} 9^{+}, \mathrm{CD} 138^{-}, \mathrm{CD}^{2} 8^{\mathrm{Low}}, \mathrm{CD}^{-}, \mathrm{IgD}^{-}$ \\
\hline \multicolumn{2}{|l|}{ T lymphocytes } \\
\hline Subset & Molecular Markers \\
\hline CD4+ & $\mathrm{CD} 19^{-}, \mathrm{CD}^{+ \text {(Mid to High) }}, \mathrm{CD}^{-}$ \\
\hline CD8+ & $\mathrm{CD}^{-} 9^{-}, \mathrm{CD}^{-}, \mathrm{CD}^{+}{ }^{+(\text {Mid to High })}$ \\
\hline Naïve & $\mathrm{CD}^{-}, \mathrm{CD}^{-}+$or $\mathrm{CD} 8+, \mathrm{CCR}^{+(\mathrm{High})}, \mathrm{CD} 45 \mathrm{RA}^{+(\text {Mid to High })}, \mathrm{CD} 45 \mathrm{RO}^{-}, \mathrm{CD} 28^{+}, \mathrm{CD}^{-} 5^{-}$ \\
\hline Stem Cell Memory & 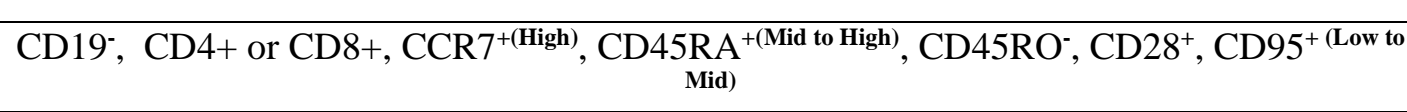 \\
\hline Central Memory & 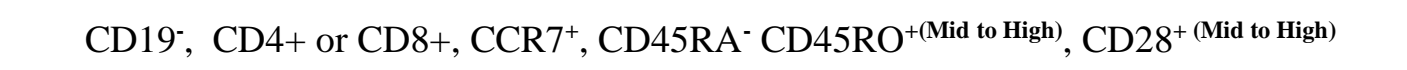 \\
\hline Transitional Memory & $\mathrm{CD}^{-}{ }^{-}, \mathrm{CD} 4+$ or $\mathrm{CD} 8+, \mathrm{CCR}^{-}, \mathrm{CD} 45 \mathrm{RA}^{-} \mathrm{CD} 45 \mathrm{RO}^{+(\mathrm{Mid})}, \mathrm{CD} 28^{+(\text {Mid to High })}$ \\
\hline Effector Memory & $\mathrm{CD}^{-} 9^{-} \mathrm{CD}^{-}+$or $\mathrm{CD} 8+, \mathrm{CCR}^{-}, \mathrm{CD}^{-} 4 \mathrm{RA}^{-} \mathrm{CD} 45 \mathrm{RO}^{+(\mathrm{Mid})}, \mathrm{CD}^{-} 8^{-}$ \\
\hline $\begin{array}{l}\text { Terminal Effector } \\
\text { Memory }\end{array}$ & $\mathrm{CD}^{-} 9^{-}, \mathrm{CD} 4+$ or $\mathrm{CD} 8+, \mathrm{CCR}^{-}, \mathrm{CD}^{-} \mathrm{RA}^{-} \mathrm{CD} 45 \mathrm{RO}^{-}, \mathrm{CD}^{-} 8^{-}$ \\
\hline TEMRA CD4+ Cells & 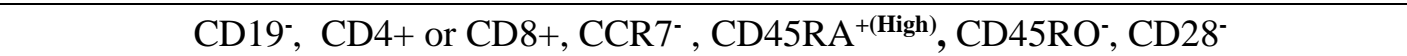 \\
\hline Tfh & CD19-, CD4+, CD8- , PD-1+, CXCR5+, CD127+, Intracellular BCL-6+ \\
\hline Treg & CD19-, CD4+, CD8- , CD25+, CD127+, Intracellular FoxP3+ \\
\hline Th17 & CD19-, CD4+, CD8- , Intracellular ROR gamma T+ \\
\hline
\end{tabular}




\section{IL-21 secretion by CD8+ central memory T cells influences both overall KSHV infection and}

\section{4 plasma cell targeting}

345 We wanted to determine whether IL-21 secretion by any particular T cell subset was correlated 346 with susceptibility to KSHV infection in our experiments. To do this, we performed B cell

347 immunophenotyping analysis to determine the extent and distribution of KSHV infection in the 348 same cultures where ICCS was performed on T cell subsets. When we examined correlations

349 between IL-21 secretion by T cell subsets and overall KSHV infection in B cells we found that

350 only IL-21+CD8+ central memory cells were significantly correlated $(r=0.57, p=0.03)$ (Fig 6A).

351 Interestingly, when we examined correlations between IL-21+ T cell subsets and the B cell subset-

352 specific distribution of KSHV infection at 3dpi, we found that the positive correlation between IL-

$35321+\mathrm{CD} 8+$ central memory $\mathrm{T}$ cells is coupled with a highly significant positive correlation to the

354 targeting of plasma cells $(\mathrm{r}=0.82, \mathrm{p}=0.0003)$ (Fig 6B \& 6C). In addition, both CD4+ and CD8+

355 IL-21+ $\mathrm{T}$ cells that express $\mathrm{RoR} \gamma \mathrm{T}+$ (the $\mathrm{Th} 17 / \mathrm{Tc} 17$-defining transcription factor) were

356 significantly correlated with plasma cell targeting by KSHV (Fig 6B \& 6D). These correlations

357 were stronger for CD4+ cells and were coupled with a negative correlation with KSHV-infection

358 of naïve T cells (Fig 6B). When we examined whether baseline (day 0) frequencies of T cell subsets

359 influenced the magnitude and distribution of KSHV infection at 3dpi we found several interesting

360 correlations (1) frequencies of CD8+ central memory cells significantly correlated with KSHV

361 targeting of plasma cell subsets (Fig 6E and 6F), (2) baseline frequencies of CD4+ RoR $\gamma \mathrm{T}+$ cells

362 significantly correlated with infection of CD20+ plasma cells (Fig 6D) and (3) both CD4+ and

363 CD8+ RoR $\gamma \mathrm{T}+$ were negatively correlated with KSHV infection of naïve B cells at 3dpi (Fig 6D).

364 Taken together, these results support a hypothetical model in which CD8+ central memory T cells

365 secrete IL-21, which supports the differentiation, survival or targeting of plasma cells, resulting in 
366 higher plasma cell infection frequencies and also an overall increase in dissemination of KSHV

367 within B lymphocytes. Our data also support the conclusion that RoR $\gamma \mathrm{T}+\mathrm{T}$ cells are participating

368 in KSHV targeting of plasma cells via IL-21 signaling, possibly by mediating differentiation of

369 naïve B cells, but this effect does not have a significant influence on overall infection in this data

370 set.

\section{Discussion}

372 Our results presented in this study indicate that KSHV can influence cytokine production in tonsil-

373 derived lymphocytes and that the host inflammatory state contributes to the dramatic variation in

374 susceptibility we observe among our tonsil lymphocyte specimens [12]. This result is not

375 surprising considering dysregulation of the inflammatory environment is a hallmark of all KSHV-

376 associated malignancies [24, 26, 27]. However, the role of the baseline inflammatory environment

377 in the oral cavity as a potentially modifiable susceptibility factor for the acquisition of KSHV

378 infection in humans is an interesting consideration stemming from these results that deserves

379 further study.

380 Our previous work demonstrated that plasma cells are highly targeted during early KSHV

381 infection, and subsequent results from our group have shown that manipulations which increase

382 plasma cell numbers also increase overall KSHV infection in B cells [28]. This latter observation

383 suggested that, in addition to being a highly targeted cell type, plasma cells are playing an active

384 role in early infection events that ultimately influences the success of initial dissemination for

385 KSHV within the B cell compartment. The results presented herein are consistent with that

386 conclusion, and mechanistically extend our understanding of how the immune microenvironment 
387 influences the establishment of infection in plasma cells. Specifically, we uncovered a critical role

388 for IL-21 signaling in this process.

389 While the precise role of IL-21 and IL-21R in human KSHV disease is poorly characterized, IL-

39021 has been studied in the pathogenesis of chronic lymphocytic choriomeningitis virus (LCMV)

391 infection, influenza virus, and, perhaps most relevant to this study, murine gammaherpesvirus 68

392 (MHV68) [22, 29, 30]. Collins and Speck recently used IL-21R knockout mice to demonstrate that

393 IL-21 signaling is critical for the establishment of MHV68 latency specifically in B cells.

394 Interestingly, this study showed that the mechanisms of decreased infection were related to

395 decreases in both germinal center and plasma cell frequencies as well as decreased infection in

396 both the germinal center and plasma cell compartment at later timepoints post-infection [22],

397 suggesting a critical mechanism for IL-21 in MHV68 transit of the germinal center and

398 differentiation of follicular-derived plasma cells. These results are generally consistent with our

399 current findings showing that IL-21 signaling promotes KSHV infection and dissemination in

400 tonsil via promotion of both plasma cell frequencies and plasma cell targeting (Fig 2\&3),

401 potentially via differentiation through a germinal center intermediate (Fig 3E). Despite this, our

402 data presented herein do not adequately address the question of whether plasma cells are

403 differentiating in response to KSHV infection and IL-21 signaling, or whether the effect we see is

404 increased survival or expansion of existing plasma cells. Studies are currently ongoing to address

405 this question more directly, and to examine the influence of viral and cellular factors on B cell

406 differentiation in our model system.

407 Moreover, both IL-21 and IL-6, which is highly induced in our KSHV-infected cultures (Fig 1),

408 are involved in the generation of RoR $\gamma \mathrm{T}+\mathrm{T}$ cells via STAT3 signaling. The resulting Th17 cells 
409 produce IL-17A which is another cytokine that promotes the establishment of chronic MHV68

410 infection via promotion of the MHV68-mediated germinal center response [31] and is

411 mechanistically linked to suppression of T cell-intrinsic IRF-1 [32]. These results are particularly

412 interesting in light of our current findings showing that IL-21 secretion from, and baseline levels

413 of, RoR $\gamma \mathrm{T}+\mathrm{T}$ cells correlate with the early targeting of plasma cells during KSHV infection (Fig

$4146 \mathrm{~B}$ and $6 \mathrm{D})$, suggesting that the Th17/Tc17 environment in the tonsil may be a critical factor

415 influencing donor-specific susceptibility to KSHV infection. Indeed, as an important site for

416 mucosal immunity in the oral cavity, the Th17/Tc17 environment in tonsil is highly dynamic and

417 physiologically important. In fact, Th17 cells play a major role in host defenses against several

418 pathogens and immunopathogenesis [33, 34]. Many studies have shown that certain parasites

419 modulate the immune response by inducing Th17 [35, 36]. Previous finding suggest that parasite

420 infection is linked with KSHV infection in Uganda [37]. Thus, the parasite burden in sub-Saharan

421 Africa may modulate susceptibility to KSHV infection via manipulating Th17/Tc17 frequencies.

422 Consistent with the MHV68 literature, our current results mechanistically implicate germinal 423 center cells in these observations. However, although our ex vivo model of KSHV infection in

424 primary lymphocytes is a powerful tool, it certainly does not recapitulate the complex interactions

425 that are needed for a functional germinal center reaction, so further examination of these particular

426 mechanisms will require the utilization of an alternative model system, such as a humanized

427 mouse. Interestingly, neither IL-21 secretion from, nor baseline levels of, CXCR5+, PD1+ TFH

428 cells correlated with increased KSHV infection or increased plasma cell targeting in our study.

429 This was surprising given that TFH are generally considered the canonical IL-21 producing cells

430 in secondary lymphoid organs [38]. This observation may indicate that IL-21 acts support plasma 
431 cell numbers during infection via an extrafollicular pathway, which is consistent with literature

432 implicating extrafollicular maturation of KSHV-infected B cells in the pathogenesis of MCD [39].

433 We observed that IL-21 secretion by CD8+ T cells with a central memory immunophenotype

434 (CCR7+CD45RO+CD28+) was significantly correlated with both overall KSHV infection and

435 plasma cell targeting, and baseline levels of CD8+ central memory $\mathrm{T}$ cells were also correlated

436 with plasma cell targeting at 3 dpi (Fig 6). Interestingly, recent studies have shown that IL-6

437 regulates IL-21 production in CD8+ T cells in a STAT3-dependent manner, and that CD8+ T cells

438 induced in this way can effectively provide help to B cells [40]. Thus, the induction of human IL-

4396 during KSHV infection may modulate the function of CD8+ T cells in a way that favors the

440 establishment and dissemination of KSHV infection within the lymphocyte compartment

441 independent of traditional CD4+ helper T cells, which would be an interesting dynamic in the

442 context of CD4+ T cell immunosuppression associated with HIV infection where KSHV-mediated

443 malignancies are common.

$445 \quad$ Material and Methods

446 Ethics Statement. Human specimens used in this research were de-identified prior to receipt, and

447 thus were not subject to IRB review as human subjects research.

448 Reagents and Cell Lines. CDw32 L cells (CRL-10680) were obtained from ATCC and were

449 cultured in DMEM supplemented with 20\% FBS (Sigma Aldrich) and Penicililin/Streptomycin/L-

450 glutamine (PSG/Corning). For preparation of feeder cells CDw32 L cells were trypsinized and

451 resuspended in $15 \mathrm{ml}$ of media in a petri dish and irradiated with 45 Gy of X-ray radiation using a

452 Rad-Source (RS200) irradiator. Irradiated cells were then counted and cyropreserved until needed 
453 for experiments. Cell-free KSHV.219 virus derived from iSLK cells [39] was a gift from Javier G.

454 Ogembo (City of Hope). Human tonsil specimens were obtained from the National Disease

455 Research Interchange (NDRI; ndriresource.org). Human fibroblasts for viral titering were derived

456 from primary human tonsil tissue and immortalized using HPV E6/E7 lentivirus derived from

457 PA317 LXSN 16E6E7 cells (ATCC CRL-2203). Antibodies for flow cytometry were from BD

458 Biosciences and Biolegend and are detailed below. Recombinant human IL-21 was from

459 Preprotech (200-21) and IL-21 neutralizing antibody was from R\&D Systems (991-R2).

460 Isolation of primary lymphocytes from human tonsils. De-identified human tonsil specimens

461 were obtained after routine tonsillectomy by NDRI and shipped overnight on wet ice in

462 DMEM+PSG. All specimens were received in the laboratory less than 24 hours post-surgery and

463 were kept at $4^{\circ} \mathrm{C}$ throughout the collection and transportation process. Lymphocytes were

464 extracted by dissection and maceration of the tissue in RPMI media. Lymphocyte-containing

465 media was passed through a $40 \mu \mathrm{m}$ filter and pelleted at $1500 \mathrm{rpm}$ for 5 minutes. RBC were lysed

466 for 5 minutes in sterile RBC lysing solution $(0.15 \mathrm{M}$ ammonium chloride, 10mM potassium

467 bicarbonate, 0.1M EDTA). After dilution to 50ml with PBS, lymphocytes were counted, and

468 pelleted. Aliquots of 5(10)7 to 1(10)8 cells were resuspended in $1 \mathrm{ml}$ of freezing media containing

$46990 \%$ FBS and 10\% DMSO and cryopreserved until needed for experiments.

$470 \quad$ Infection of primary lymphocytes with $\mathbf{K S H} \boldsymbol{V}$. Lymphocytes were thawed rapidly at $37^{\circ} \mathrm{C}$, diluted

471 dropwise to $5 \mathrm{ml}$ with RPMI and pelleted. Pellets were resuspended in $1 \mathrm{ml}$

$472 \mathrm{RPMI}+20 \% \mathrm{FBS}+100 \mu \mathrm{g} / \mathrm{ml}$ DNaseI+ Primocin $100 \mu \mathrm{g} / \mathrm{ml}$ and allowed to recover in a low-binding

47324 well plate for 2 hours at $37^{\circ} \mathrm{C}, 5 \% \mathrm{CO} 2$. After recovery, total lymphocytes were counted and

474 naïve B cells were isolated using Mojosort Naïve B cell isolation beads (Biolegend 480068) or

475 Naïve B cell Isolation Kit II (Miltenyi 130-091-150) according to manufacturer instructions. 
476 Bound cells (non-naïve B and other lymphocytes) were retained and kept at $37^{\circ} \mathrm{C}$ in $\mathrm{RPMI}+20 \%$

477 FBS+ Primocin $100 \mu \mathrm{g} / \mathrm{ml}$ during the initial infection process. $1(10)^{6}$ Isolated naïve B cells were

478 infected with iSLK-derived KSHV.219 (dose equivalent to the ID20 at 3dpi on human fibroblasts)

479 or Mock infected in 400ul of total of virus + serum free RPMI in $12 \times 75 \mathrm{~mm}$ round bottom tubes

480 via spinoculation at $1000 \mathrm{rpm}$ for 30 minutes at $4^{\circ} \mathrm{C}$ followed by incubation at $37^{\circ} \mathrm{C}$ for an

481 additional 30 minutes. Following infection, cells were plated on irradiated CDW32 feeder cells in

482 a 48 well plate, reserved bound cell fractions were added back to the infected cell cultures, and

483 FBS and Primocin (Invivogen) were added to final concentrations of $20 \%$ and $100 \mu \mathrm{g} / \mathrm{ml}$,

484 respectively and recombinant cytokines or neutralizing antibodies were also added at this stage,

485 depending upon the specific experiment. Cultures were incubated at $37^{\circ} \mathrm{C}, 5 \% \mathrm{CO} 2$ for the

486 duration of the experiment. At 3 days post-infection, cells were harvested for analysis by flow

487 cytometry and supernatants were harvested, clarified by centrifugation for 15 minutes at 15,000

$488 \mathrm{rpm}$ to remove cellular debris, and stored at $-80^{\circ} \mathrm{C}$ for analysis.

Bead-based immunoassay for supernatant cytokines. Clarified supernatants were thawed on ice

490 and $25 \mu 1$ of each was added to a 13-plex LEGENDplex (Biolegend) bead-based immunoassay

491 containing capture beads for the following analytes: IL-5, IL-13, IL-2, IL-9, IL-10, IL17A, IL-

$49217 \mathrm{~F}, \mathrm{IL}-6$, IL-21, IL-22, IL-4, TNF- $\alpha$, and IFN- $\gamma$. These assays were performed according to the

493 manufacturer's instructions, data was acquired for 5000 beads per sample (based on approximately

494300 beads per analyte recommended by the manufacturer) using a BD FACS VERSE flow

495 cytometry analyzer and cytokine concentrations in the experimental supernatants was calculated

496 from standard curves using the LEGENDPlex software. 

PBS containing zombie violet fixable viability stain (BL Cat\# 423113) and incubated on ice for 15 minutes. After incubation, cells were pelleted and resuspended in 100ul PBS, containing the following: $2 \% \mathrm{FBS}$ and $0.5 \%$ BSA (FACS Block) was added to the wells. Cells were pelleted at 1500rpm 5 minutes and resuspended in 200ul FACS Block for 10 minutes on ice. Cells were pelleted at $1500 \mathrm{rpm}$ for 5 minutes and resuspended in $50 \mu \mathrm{l}$ of PBS with $0.5 \%$ BSA and $0.1 \%$ Sodium Azide (FACS Wash), For B cell frequencies $10 \mu \mathrm{l}$ BD Brilliant Stain Buffer Plus and antibodies as follows: IgD-BUV395 (2.5 $\mu 1 /$ test BD 563823), CD77-BV510 (2.0 $\mu \mathrm{l} /$ test BD

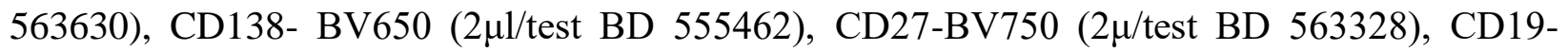
PerCPCy5.5 (2.0 $\mu 1 /$ test BD 561295), CD38-APC (10 $\mu \mathrm{l} /$ test BD 560158), CD20-APCH7 (2ul/test

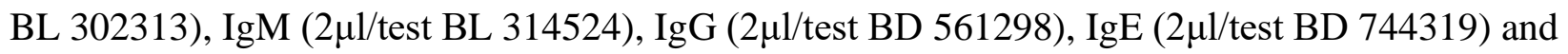

511 frequencies $0.5(10)^{6}$ cells from baseline uninfected total lymphocyte samples were stained and 512 analyzed as above with phenotype antibody panel as follows: CD95-APC (2 $\mu 1$, Biolegend

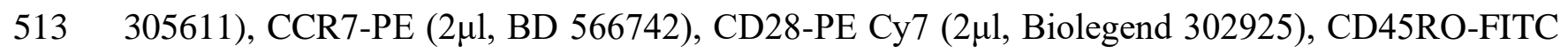

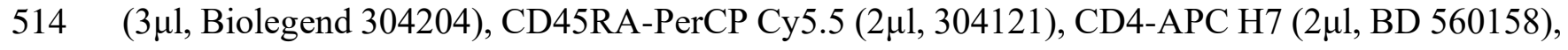

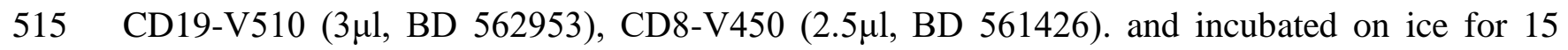
516 minutes. After incubation, $150 \mu \mathrm{l}$ FACS Wash was added. Cells were pelleted at 1500rpm for 5

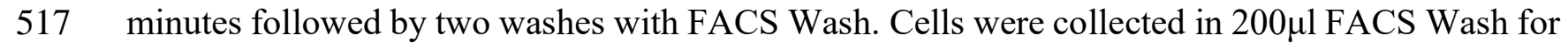
518 flow cytometry analysis. Cells were analyzed using an LSR Fortessa X-20 cell analyzer (BD 519 Biosciences). BD CompBeads (51-90-9001229) were used to calculate compensation for all 
520 antibody stains and methanol-fixed Namalwa cells (ATCC CRL1432) +/- KSHV were used to

521 calculate compensation for GFP and the fixable viability stain. Flow cytometry data was analyzed

522 using FlowJo software and exported for quantitative analysis in R as described below.

523 ICCS for IL-21 secretion. At 3dpi, cultures were treated for 6 hours with [4 ul for every $6 \mathrm{ml}$ of

524 cel culture] monensin to block cytokine secretion. Following incubation, approximately 1 million

525 cells were harvested and viability and surface staining for T cell lineage markers was performed

526 as described above. After the final wash, cells were fixed for 10 minutes in BD cytofix/cytoperm

527 (51-2090KZ), pelleted and further treated for 10 minutes with cytofix/cytoperm+10\% DMSO

528 (superperm) to more effectively get intracellular antibodies into the nucleus. Intracellular

529 antibodies, as follows, were diluted in 1x BD Permwash (51-2091KZ) and left on fixed cells

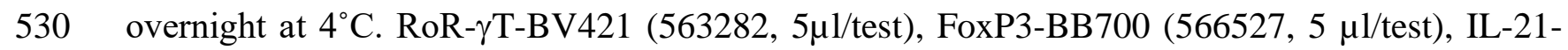

531 APC (513007, $5 \mu 1 /$ test), BLC6-BV711 (561080, 5 $\mu 1 /$ test). Cells were then washed twice with 1x

532 permwash and analyzed as described above.

$533 \boldsymbol{R T}$-PCR. At 3 days post infection, $1(10)^{6}$ lymphocytes were harvested into an equal volume of

534 Trizol and DNA/RNA shield (Zymo Research R110-250). Total RNA was extracted using using

535 Zymo Directzol Microprep (Zymo Research R2060) according to manufacturer instructions. RNA

536 was eluted in $10 \mu \mathrm{H} 2 \mathrm{O}$ containing $2 \mathrm{U}$ RNase inhibitors and a second DNase step was performed

537 for 30 minutes using the Turbo DNA-Free kit (Invitrogen AM1907M) according to manufacturer

538 instructions. One-step RT-PCR cDNA synthesis and preamplification of GAPDH, LANA and

539 K8.1 transcripts was performed on 15ng of total RNA using the Superscript III One-step RT-PCR

540 kit (ThermoFisher 12574026). 
541 Duplicate no RT (NRT) control reactions were assembled for each sample containing only

542 Platinum Taq DNA polymerase (Thermofisher 15966005) instead of the Superscript III RT/Taq

543 DNA polymerase mix. After cDNA synthesis and 20 cycles of target pre-amplification, $2 \mu 1$ of pre-

544 amplified cDNA or NRT control reaction was used as template for multiplexed real-time PCR

545 reactions using TaqProbe 5x qPCR MasterMix -Multiplex (ABM MasterMix-5PM), 5\% DMSO,

546 primers at $900 \mathrm{nM}$ and probes at 250nM against target genes. All primer and probe sequences used

547 in these assays have been previously published [12]. Real time PCR was performed using a 40-

548 cycle program on a Biorad real time thermocycler. Data is represented as quantitation cycle $(\mathrm{Cq})$

549 and assays in which there was no detectable $\mathrm{Cq}$ value were set numerically as $\mathrm{Cq}=41$ for analysis

550 and data visualization. The expression of each gene was normalized to that of a housekeeping

551 gene GAPDH.

552 Statistical Analysis. The indicated data sets and statistical analysis were performed in Rstudio

553 software using ggplot2 [41], ggcorrplo [42], and tidyverse [43] packages. Statistical analysis was

554 performed using rstatix [44] package. Specific methods of statistical analysis including Anova,

555 independent t-test and Pearson correlations and resulting values for significance and correlation

556 are detailed in the corresponding figure legends.

558 Figure Legends

559 Figure 1: KSHV alters cytokine secretion and cytokines affect the establishment of infection.

56033 replicate infections using 24 unique tonsil specimens were performed using KSHV.219

561 infection of naïve B lymphocytes followed by reconstitution of the total lymphocyte environment

562 and culture on CDW32 feeder cells. At 3 dpi, cells were collected for flow cytometry analysis for 
563 infection (GFP) and B cell subsets using our previously-characterized immunophenotyping panel

564 and supernatants were collected for analysis of cytokines by multiplex immunoassay (Biolegend

565 Legendplex) (A) cytokine production in Mock and KSHV-infected cultures showing individual

566 sample quantities and means (red diamonds, top panels) and matched Mock and KSHV samples

567 to show trends of induction/repression (bottom panels) Statistical analysis was performed by one-

568 way repeated measures ANOVA. $\mathrm{p}=0.01 \mathrm{~F}=7.06$ for IL-5, $\mathrm{p}=0.0001 \mathrm{~F}=14$ for IL-6, $\mathrm{p}=0.5 \mathrm{~F}=4.3$

569 for IL-4 (B) Data as in (A) showing the level of induction or repression of each cytokine

570 comparing KSHV to matched Mock cultures (C) Induction or repression of all cytokines (left y-

571 axis) on a per-sample basis ordered based on overall susceptibility based on percentage of GFP+

572 B lymphocytes in the same culture (right y-axis, red diamonds) (D) Pairwise correlations using

573 Pearson method between cytokine concentration (y-axis) and overall infection (x-axis) in KSHV-

574 infected lymphocyte cultures (E) Pairwise correlations using Pearson method between cytokine

575 concentration (y-axis) and Percent GFP+ within CD138+ (x-axis) in KSHV-infected lymphocyte

576 cultures. For panels A, B, D and E colors indicate individual tonsil specimens and can be compared

577 between panels.

Figure 2: IL-21 supplementation increases overall KSHV infection and plasma cell

frequencies. Lymphocytes from 12 tonsil donors were infected with KSHV.219 and cultured with

581 indicated doses of recombinant human IL-21 and analyzed at 3dpi by flow cytometry (A) the dose

582 effect of IL-21 supplementation on GFP+ viable B lymphocytes. (B) data as in (A) normalized to

583 the untreated control for each specimen. (C) 14 additional tonsil donors analyzed as in (A) with

584 only $100 \mathrm{pg} / \mathrm{ml} \mathrm{IL-21} \mathrm{treatment.} \mathrm{Red} \mathrm{diamonds} \mathrm{indicate} \mathrm{group} \mathrm{means.} \mathrm{p}=0.02, \mathrm{~F}=6.4$ via one-way

585 repeated measures ANOVA. (D) tonsil lymphocyte specimens from (C) were stained for B cell 
immunophenotypes and analyzed by flow cytometry. Red boxes indicate subsets with significant repeated measures ANOVA analysis. See Table 1 for $\mathrm{p}$-values and F statistics from this analysis. (E) immunophenotyping data was used to determine the within-subset frequency of GFP+ cells in the KSHV-infected conditions with or without IL-21 treatment. Top panels show individual

591 sample quantities and means (red diamonds) and bottom panels show trends of increased/decreased subset targeting on a per-sample basis. Colored points denote unique tonsil

593 specimens and can be compared between panels D and E. (F) Pearson correlation between overall

594 GFP+ B cells in KSHV-infected, IL-21 treated cultures as in (C) and the level of plasma cells 595 (right) and infection of plasma cells (left). Blue line is linear model regression and grey shading 596 indicates $95 \%$ confidence interval.

Figure 3: IL-21 neutralization inhibits the establishment of KSHV infection. Naïve B cells

598 from 11 unique tonsil specimens were Mock or KSHV-infected and indicated concentrations of IL-21 neutralizing antibody was added to the resulting total lymphocyte cultures. Cultures were

600 analyzed at 3 dpi for B lymphocyte immunophenotypes and the distribution of KSHV infection 601 via GFP expression. Total GFP+ viable B lymphocytes represented as (A) raw percentages or (B) 602 normalized to the untreated control for each tonsil sample. For (A) one-way repeated measures 603 ANOVA shows $\mathrm{p}=0.00001, \mathrm{~F}=9.4$ for the main effect of IL-21 neutralization and Dunnett's test 604 reveals $\mathrm{p}=0.03$ at the $100 \mu \mathrm{g} / \mathrm{ml}$ dose. (C) frequencies of B cell subsets in the cultures. Red boxes 605 indicate significant results as follows: Two-way repeated measures ANOVA on the raw data 606 showed significant effects of IL-21 treatment on B subset frequencies; IgG+ $(\mathrm{p}=0.04, F=5.0)$, 607 memory $(\mathrm{p}=0.03, \mathrm{~F}=3.4)$, and double negative $(\mathrm{p}=0.02, \mathrm{~F}=3.8), \operatorname{IgM}+(\mathrm{p}=0.0003, \mathrm{~F}=8.6)$, 608 transitional $(\mathrm{p}=0.01, \mathrm{~F}=4.1)$. Post-hoc paired T-tests showed significant effect of $200 \mu \mathrm{g} / \mathrm{ml}$ 
neutralizing antibody on total plasma cells $(\mathrm{p}=0.02)$ and CD20+ plasma cells $(\mathrm{p}=0.005)$ in Mock

610 cultures only. (D) Effect of indicated doses of IL-21 neutralizing antibody on KSHV infection of

611 B cell subsets. Data is normalized to each sample's untreated control level for each B cell subset.

612 One-way repeated measures ANOVA on the raw data reveals a significant effect on infection of

613 transitional B cells $(\mathrm{p}=0.01, \mathrm{~F}=4.0)$. (E) Correlation between the effect of IL-21 neutralization on

614 overall infection (x-axis) and the contribution of each subset to KSHV infection in the same

615 condition (y-axis). Shapes indicate doses in this panel (circle $=100 \mu \mathrm{g} / \mathrm{ml}$, triangle $=200 \mu \mathrm{g} / \mathrm{ml}$,

616 square $=400 \mu \mathrm{g} / \mathrm{ml})$. Subsets with statistically significant correlations are indicated by red boxes

617 with statistics from Pearson's linear correlation as follows: CD20+ plasma cells $(r=0.6, p=0.0002)$,

618 plasma cells $(\mathrm{r}=0.6, \mathrm{p}=0.0003)$, transitional $(\mathrm{r}=0.5, \mathrm{p}=0.004)$, germinal center $(\mathrm{r}=-0.4, \mathrm{p}=0.02)$.

619 For panels C-E colors indicate unique tonsil specimens and can be compared between these panels

620 and red diamonds indicate the mean value for all tonsil specimens. RT-PCR analysis of KSHV

621 transcripts at $3 \mathrm{dpi}$ in 8 unique tonsil specimens with either IL-21 supplementation at $100 \mathrm{pg} / \mathrm{ml}$

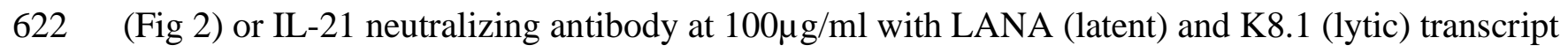

623 targets. No RT controls were used to determine that RT-PCR signal is not due to DNA

624 contamination (F) Cq values for viral targets normalized to the within-sample Cq for GAPDH (G)

625 GAPDH-normalized values further normalized to the within-sample value for the untreated

626 control.

627 Figure 4: IL21 receptor distribution in primary human tonsil B lymphocytes affects the

628 magnitude and distribution of KSHV infection. B cell immunophenotyping analysis including

629 IL-21R was performed at baseline (Day 0) for 10 unique tonsil specimens. (A) total percentage of

630 IL-21R+ within viable CD19+ B cells. (B) Percent of individual B cell subsets within IL-21+ B

631 cells. Red diamonds indicate the mean value for all tonsil specimens and (C) distribution of B cell 
632 subsets within IL-21+ on a per-tonsil basis. (D) correlation analysis of baseline IL-21 receptor

633 distribution within subsets and the overall susceptibility of the same tonsil specimen to KSHV

634 infection at 3 dpi. Pearson correlation coefficients and p-values are denoted on panels where the

635 result is statistically significant. (E) correlation analysis of baseline IL-21 receptor distribution

636 with the effect of IL-21 supplementation on overall KSHV infection in the same tonsil specimens.

637 Pearson correlation coefficients and p-values are denoted on panels where the result is statistically

638 significant. (F) correlation analysis of baseline IL-21 receptor distribution with the IL-21 mediated

639 increase in total plasma cell numbers at 3dpi in KSHV-infected cultures in the same tonsil

640 specimens. (G) Distribution of IL-21 receptor at day 0 (baseline) or 3dpi within Mock,

641 Mock+100ng/ml IL-21, KSHV or KSHV+ 100ng/ml IL-21 conditions. Red diamonds indicate the

642 mean values for each condition and significant differences were assessed via paired T-test.

\section{Figure 5: Characterization of $\mathbf{T}$ cell subsets producing IL-21 in primary human tonsil B}

644 lymphocytes. T cells were analyzed by surface immunophenotyping, intracellular transcription

645 factor staining and ICCS for IL-21 secretion in Mock and KSHV-infected total lymphocyte

646 cultures at 3 dpi in 14 unique tonsil specimens. (A) Total IL-21+ viable non-B cells (B) percent of

647 CD4+ or CD8+ T cells within IL-21+. For (A) and (B) red diamonds indicate the mean value for

648 the condition and colors indicate specific tonsil specimens and can be compared between the

649 panels. (C) Immunophenotypic analysis of T cell subsets (Table 2) within IL-21+ in Mock (red)

650 and KSHV (blue) cultures. (D) Data as in (C) but normalized to show the induction or repression

651 of the $\mathrm{T}$ cell subset within IL-21+ cells (KSHV-Mock). ${ }^{*} \mathrm{p}=0.05 ; * * \mathrm{p}=0.04 ; * * * \mathrm{p}=0.003$

652 comparing Mock and KSHV-infected via paired T test pre-normalization. 


\section{Figure 6: IL-21 secretion by CD8+ central memory $\mathrm{T}$ cells influences both overall KSHV}

654 infection and plasma cell targeting. Lymphocyte cultures from the experiments shown in Fig 5

655 were further analyzed for B cell subsets and the magnitude and distribution of KSHV infection.

656 (A) correlation analysis of total GFP+ within viable, CD19+ B lymphocytes at 3 dpi with total IL-

65721 secretion in non-B cells or the contribution of individual $\mathrm{T}$ cell subsets to IL-21 secretion.

658 Pearson's correlation coefficient and p-value is shown on panels that have statistically significant

659 correlations. (B) correlogram of Pearson correlations between the distribution of KSHV infection

660 within B cell subsets (y-axis) and total IL-21 or the contribution of individual T cell subsets to IL-

66121 secretion (x-axis). Pearson's $r$ values with an absolute value greater than or equal to 0.53 are

662 statistically significant for this dataset. (C) scatterplot and linear model fit for the correlation

663 between GFP+ plasma cells and IL-21 secretion by CD8+ central memory T cells (D) Scatterplots

664 and linear model fits for the correlations between GFP+ plasma cells and secretion of IL-21 by

665 RoR $\gamma \mathrm{T}+\mathrm{CD} 4+(\mathrm{left})$ or CD8+ (right) T cells. (E) correlogram of Pearson correlations between the

666 distribution of KSHV infection within B cell subsets (y-axis) and baseline (Day 0) levels of T cell

667 subsets. Pearson's r values with an absolute value greater than or equal to 0.53 are statistically

668 significant for this dataset. (F) scatterplot and linear model fit for the correlation between GFP+

669 plasma cells and baseline frequency of CD8+ central memory T cells. For A, C, D and F grey

670 shading indicates $95 \%$ confidence intervals.

671 Supplemental Figure 1: Representative gating scheme for $\mathrm{T}$ cell immunophenotyping using

672 lineage definitions as detailed in Table 2.

673 Supplemental Figure 2: Total T cell subset frequencies in Mock and KSHV-infected cultures as

674 in Figure 5. 


\section{References}

677 1. Chang, Y., et al., Identification of herpesvirus-like DNA sequences in AIDS-associated 678 Kaposi's sarcoma. Science, 1994. 266(5192): p. 1865-1869.

679 2. Ablashi, D.V., et al., Spectrum of Kaposi's sarcoma-associated herpesvirus, or human 680 herpesvirus 8, diseases. Clinical microbiology reviews, 2002. 15(3): p. 439-464.

$6813 . \quad$ Cesarman, E., et al., Kaposi's sarcoma-associated herpesvirus-like DNA sequences in

$682 \quad$ AIDS-related body-cavity-based lymphomas. New England Journal of Medicine, 1995. 332(18): p. 1186-1191.

4. Soulier, J., et al., Kaposi's sarcoma-associated herpesvirus-like DNA sequences in multicentric Castleman's disease [see comments]. Blood, 1995. 86(4): p. 1276-1280.

5. Uldrick, T.S., et al., An interleukin-6-related systemic inflammatory syndrome in patients co-infected with Kaposi sarcoma-associated herpesvirus and HIV but without Multicentric Castleman disease. Clinical Infectious Diseases, 2010. 51(3): p. 350-358.

6. Bouvard, V., et al., A review of human carcinogens--Part B: biological agents. The Lancet. Oncology, 2009. 10(4): p. 321-322.

7. $\quad$ Parkin, D.M., The global health burden of infection-associated cancers in the year 2002. International journal of cancer, 2006. 118(12): p. 3030-3044.

8. Ganem, D., KSHV and the pathogenesis of Kaposi sarcoma: listening to human biology and medicine. The Journal of clinical investigation, 2010. 120(4): p. 939-949.

9. Casper, C., et al., Frequent and asymptomatic oropharyngeal shedding of human herpesvirus 8 among immunocompetent men. The Journal of infectious diseases, 2007. 195(1): p. 30-36.

10. Alomari, N. and J. Totonchy, Cytokine-Targeted Therapeutics for KSHV-Associated Disease. Viruses, 2020. 12(10): p. 1097.

11. Carbone, A., et al., Understanding pathogenetic aspects and clinical presentation of primary effusion lymphoma (PEL) through its derived cell lines. AIDS (London, England), 2010. 24(4): p. 479.

12. Aalam, F., et al., Analysis of KSHV B lymphocyte lineage tropism in human tonsil reveals efficient infection of CD138+ plasma cells. PLoS pathogens, 2020. 16(10): p. e1008968.

13. Parrish-Novak, J., et al., Interleukin-21 and the IL-21 receptor: novel effectors of NK and $T$ cell responses. Journal of leukocyte biology, 2002. 72(5): p. 856-863.

14. Parrish-Novak, J., et al., Interleukin 21 and its receptor are involved in NK cell expansion and regulation of lymphocyte function. Nature, 2000. 408(6808): p. 57-63.

15. Kuchen, S., et al., Essential role of IL-21 in B cell activation, expansion, and plasma cell generation during CD4+ T cell-B cell collaboration. The journal of immunology, 2007. 179(9): p. 5886-5896.

16. Konforte, D., N. Simard, and C.J. Paige, IL-21: an executor of B cell fate. The Journal of Immunology, 2009. 182(4): p. 1781-1787.

17. Ozaki, K., et al., Regulation of B cell differentiation and plasma cell generation by IL-21, a novel inducer of Blimp-1 and Bcl-6. The Journal of Immunology, 2004. 173(9): p. 5361-5371. 
18. Calame, K.L., K.-I. Lin, and C. Tunyaplin, Regulatory mechanisms that determine the development and function of plasma cells. Annual review of immunology, 2003. 21(1): p. 205-230.

19. Yajima, H., et al., Loss of interleukin-21 leads to atrophic germinal centers in

20. Konforte, D. and C.J. Paige, Identification of cellular intermediates and molecular pathways induced by IL-21 in human B cells. The Journal of Immunology, 2006. 177(12): p. 8381-8392. Epstein-Barr virus oncoproteins, EBNA2 and LMP1, in infected human B cells. Virology, 2008. 374(1): p. 100-113.

22. Collins, C.M. and S.H. Speck, Interleukin 21 signaling in B cells is required for efficient establishment of murine gammaherpesvirus latency. PLoS pathogens, 2015. 11(4): p. e1004831.

23. Gasperini, P. and G. Tosato, Targeting the mammalian target of Rapamycin to inhibit VEGF and cytokines for the treatment of primary effusion lymphoma. Leukemia, 2009. 23(10): p. 1867-1874.

24. Gasperini, P., S. Sakakibara, and G. Tosato, Contribution of viral and cellular cytokines to Kaposi's sarcoma-associated herpesvirus pathogenesis. Journal of leukocyte biology, 2008. 84(4): p. 994-1000.

25. Totonchy, J., et al., KSHV induces immunoglobulin rearrangements in mature $B$ lymphocytes. PLoS pathogens, 2018. 14(4): p. e1006967.

26. Chang, J., et al., Induction of Kaposi's sarcoma-associated herpesvirus from latency by inflammatory cytokines. Virology, 2000. 266: p. 17-25.

27. Chang, J., et al., Inflammatory cytokines and the reactivation of Kaposi's sarcomaassociated herpesvirus lytic replication. Virology, 2000. 266(1): p. 17-25.

28. Palmerin, N., et al., Suppression of DC-SIGN and gH Reveals Complex, Subset-Specific Mechanisms for KSHV Entry in Primary B Lymphocytes. Viruses 2021, 13, 1512. 2021, s Note: MDPI stays neutral with regard to jurisdictional claims in published ....

29. Schmitz, I., et al., IL-21 restricts virus-driven Treg cell expansion in chronic LCMV infection. PLoS pathogens, 2013. 9(5): p. e1003362.

30. Pallikkuth, S., et al., Upregulation of IL-21 receptor on B cells and IL-21 secretion distinguishes novel $2009 \mathrm{H} 1 \mathrm{N1}$ vaccine responders from nonresponders among HIVinfected persons on combination antiretroviral therapy. The Journal of Immunology, 2011. 186(11): p. 6173-6181.

31. Jondle, C., et al., Gammaherpesvirus Usurps Host IL-17 Signaling To Support the Establishment of Chronic Infection. Mbio, 2021. 12(2): p. e00566-21.

32. Jondle, C., et al., T Cell-Intrinsic Interferon Regulatory Factor 1 Expression Suppresses Differentiation of CD4+ T Cell Populations That Support Chronic Gammaherpesvirus Infection. Journal of Virology, 2021. 95(20): p. e00726-21.

33. Gaddi, P.J. and G.S. Yap, Cytokine regulation of immunopathology in toxoplasmosis. Immunology and cell biology, 2007. 85(2): p. 155-159.

34. Kelly, M.N., et al., Interleukin-17/interleukin-17 receptor-mediated signaling is important for generation of an optimal polymorphonuclear response against Toxoplasma gondii infection. Infection and immunity, 2005. 73(1): p. 617-621. 
35. Shainheit, M.G., et al., The pathogenic Th17 cell response to major schistosome egg antigen is sequentially dependent on IL-23 and IL-1 $\beta$. The Journal of Immunology, 2011. 187(10): p. 5328-5335.

36. Wen, X., et al., Dynamics of Th17 cells and their role in Schistosoma japonicum infection in C57BL/6 mice. PLoS neglected tropical diseases, 2011. 5(11): p. e1399.

37. Wakeham, K., et al., Parasite infection is associated with Kaposi's sarcoma associated herpesvirus (KSHV) in Ugandan women. Infectious agents and cancer, 2011. 6(1): p. 1-7.

38. Song, W. and J. Craft, T follicular helper cell heterogeneity: time, space, and function. Immunological reviews, 2019. 288(1): p. 85-96.

39. Totonchy, J., Extrafollicular activities: perspectives on HIV infection, germinal centerindependent maturation pathways, and KSHV-mediated lymphoproliferation. Current opinion in virology, 2017. 26: p. 69-73.

40. Yang, R., et al., IL-6 promotes the differentiation of a subset of naive CD8+T cells into IL-21-producing B helper CD8+ T cells. Journal of Experimental Medicine, 2016. 213(11): p. 2281-2291.

41. Wickham, H., Ggplot2 : elegant graphics for data analysis. Use R! 2009, New York: Springer. viii, $212 \mathrm{p}$.

42. Kassambara, A., ggcorrplot: Visualization of a Correlation Matrix using 'ggplot2'. 2019.

43. Lander, J.P., $R$ for everyone : advanced analytics and graphics. Second edition ed. The Addison Wesley data and anlytics series. 2017, Boston: Addison-Wesley. xxiv, 528 pages.

44. Kassambara, A., rstatix: Pipe-Friendly Framework for Basic Statistical Tests. 2020. 


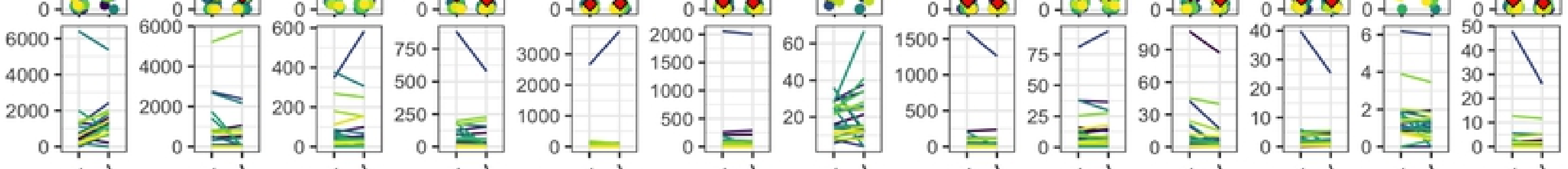

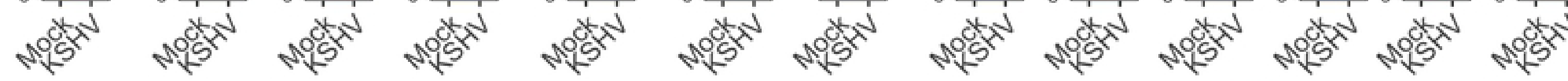

B

B

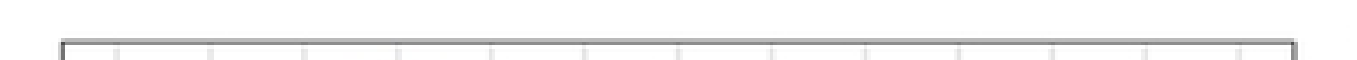
C - HL.5 $\bullet$ IL.6 $\bullet$ IFNg $\bullet$ LL.17F $\bullet$ L.22

- IL.13 - IL.9 - TNFa

- IL.2 - IL.10 $\bullet$ IL.17A $\bullet$ IL.21
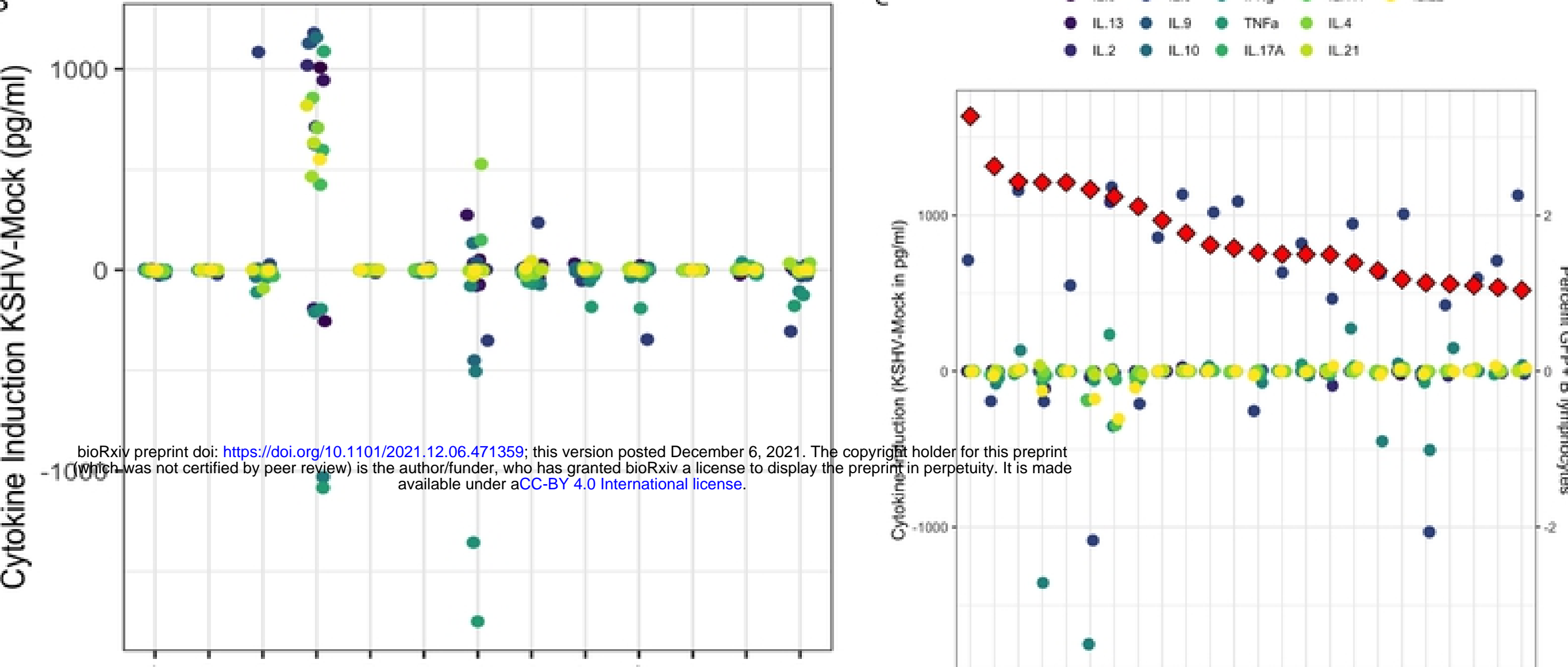

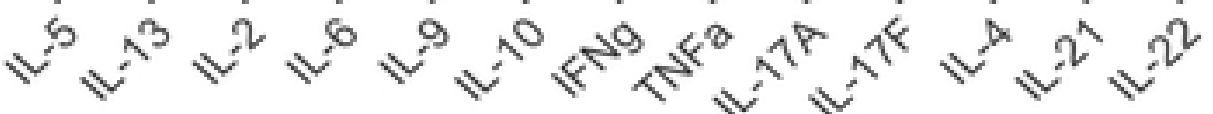

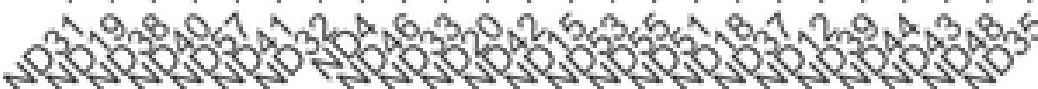
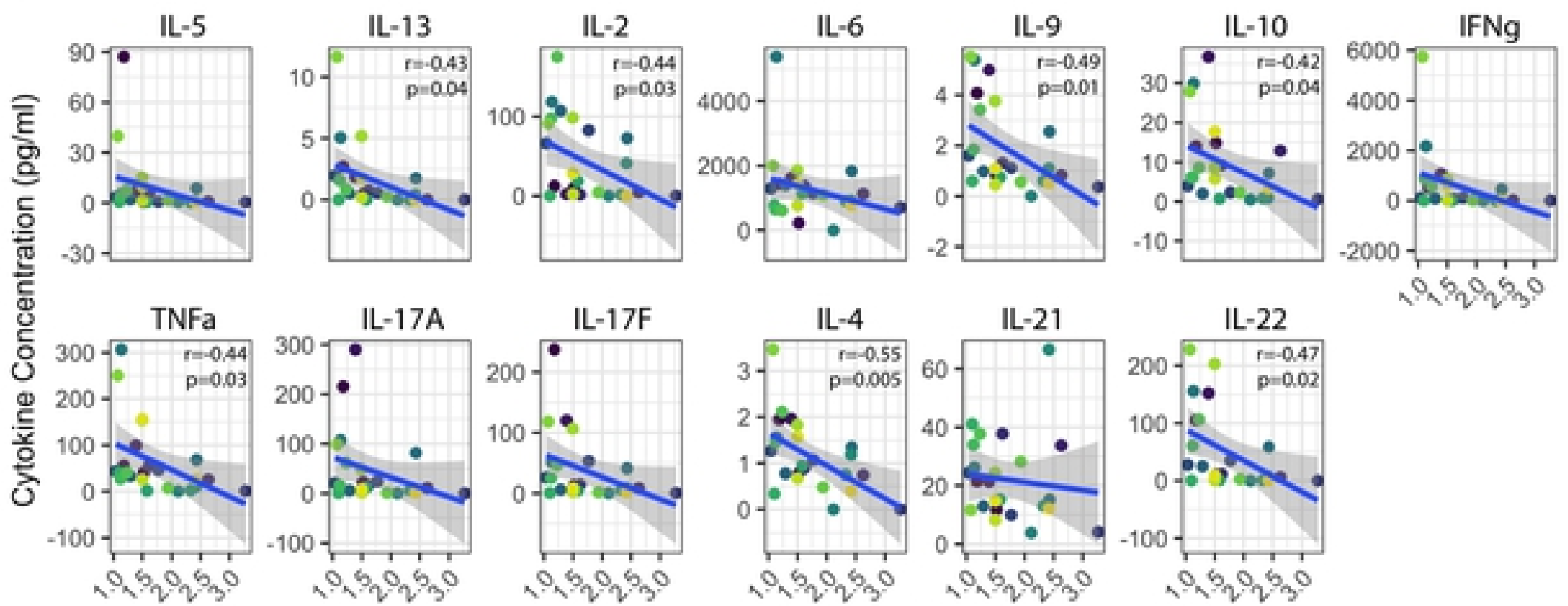

$0,522^{2} 30$

Percent GFP+ Viable B cells
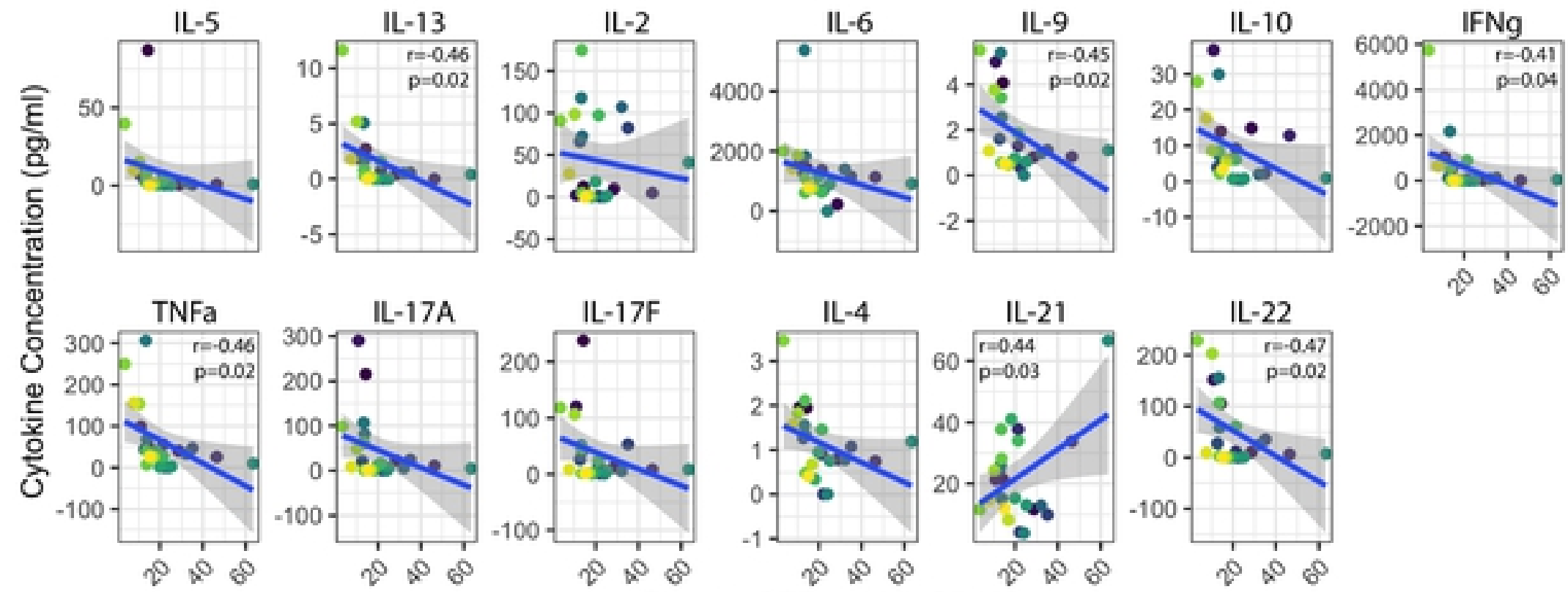

Percent GFP+ within CD138+

Figure 1 

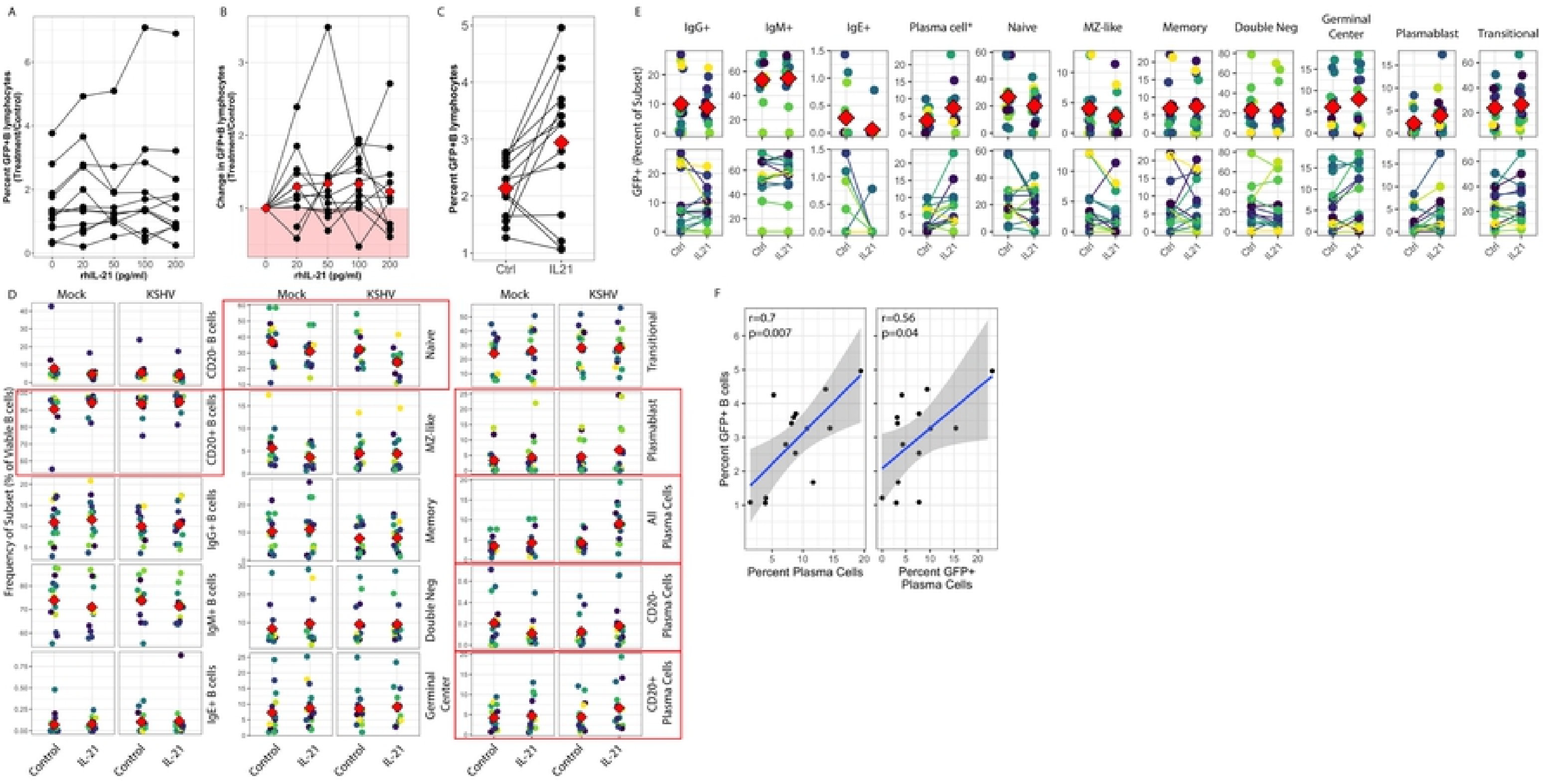

Figure 2 

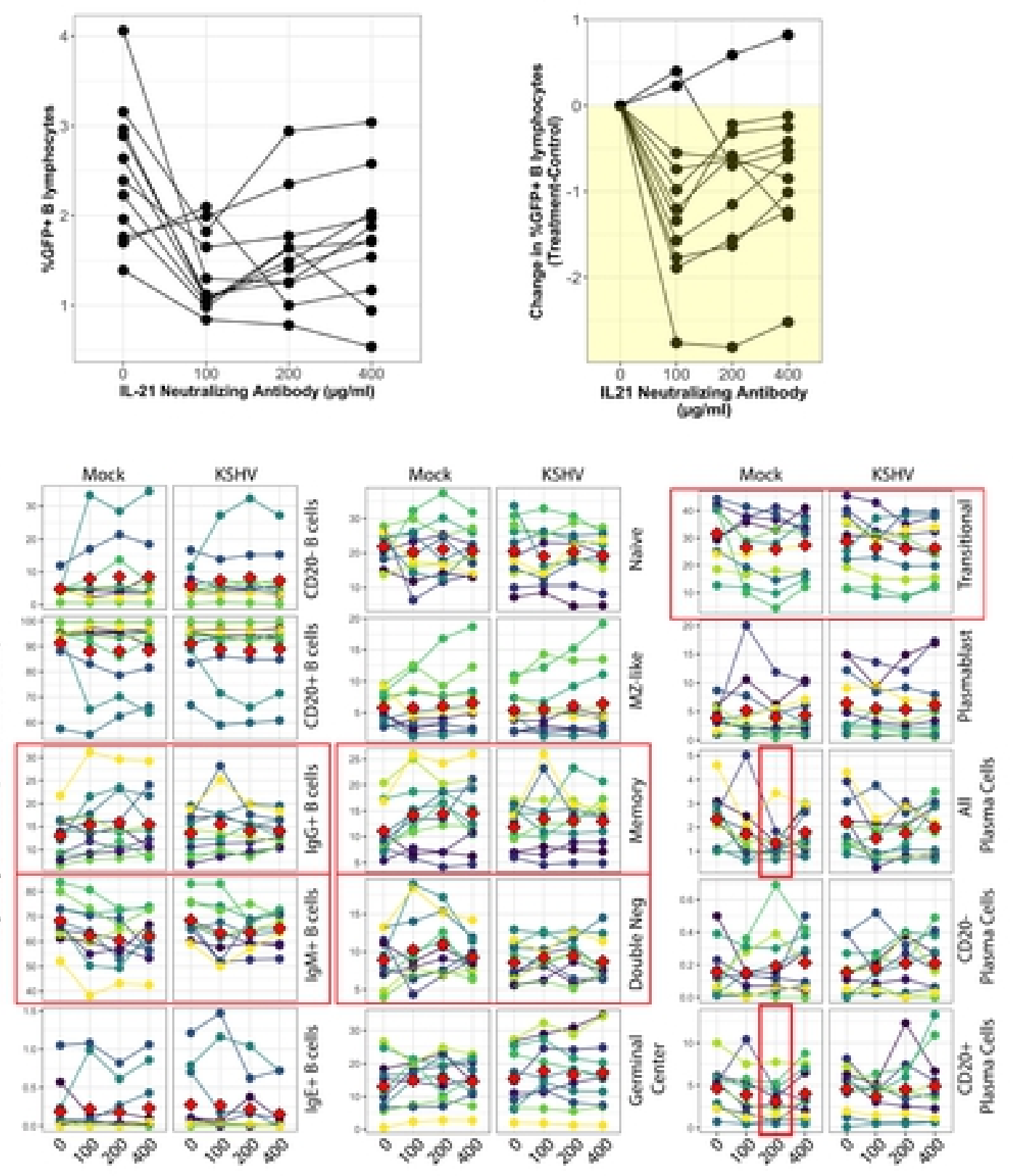

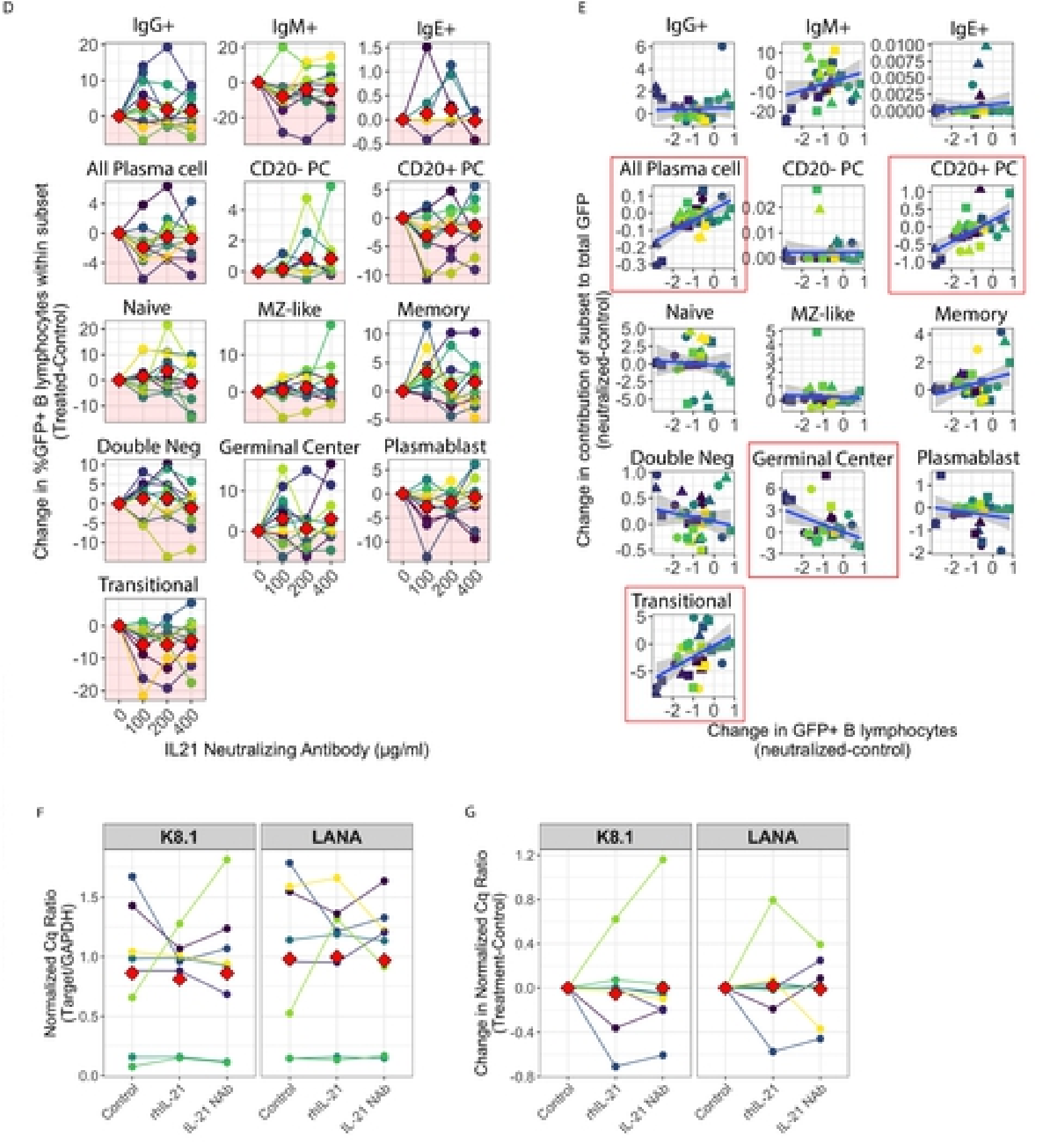

\section{Figure 3}


B
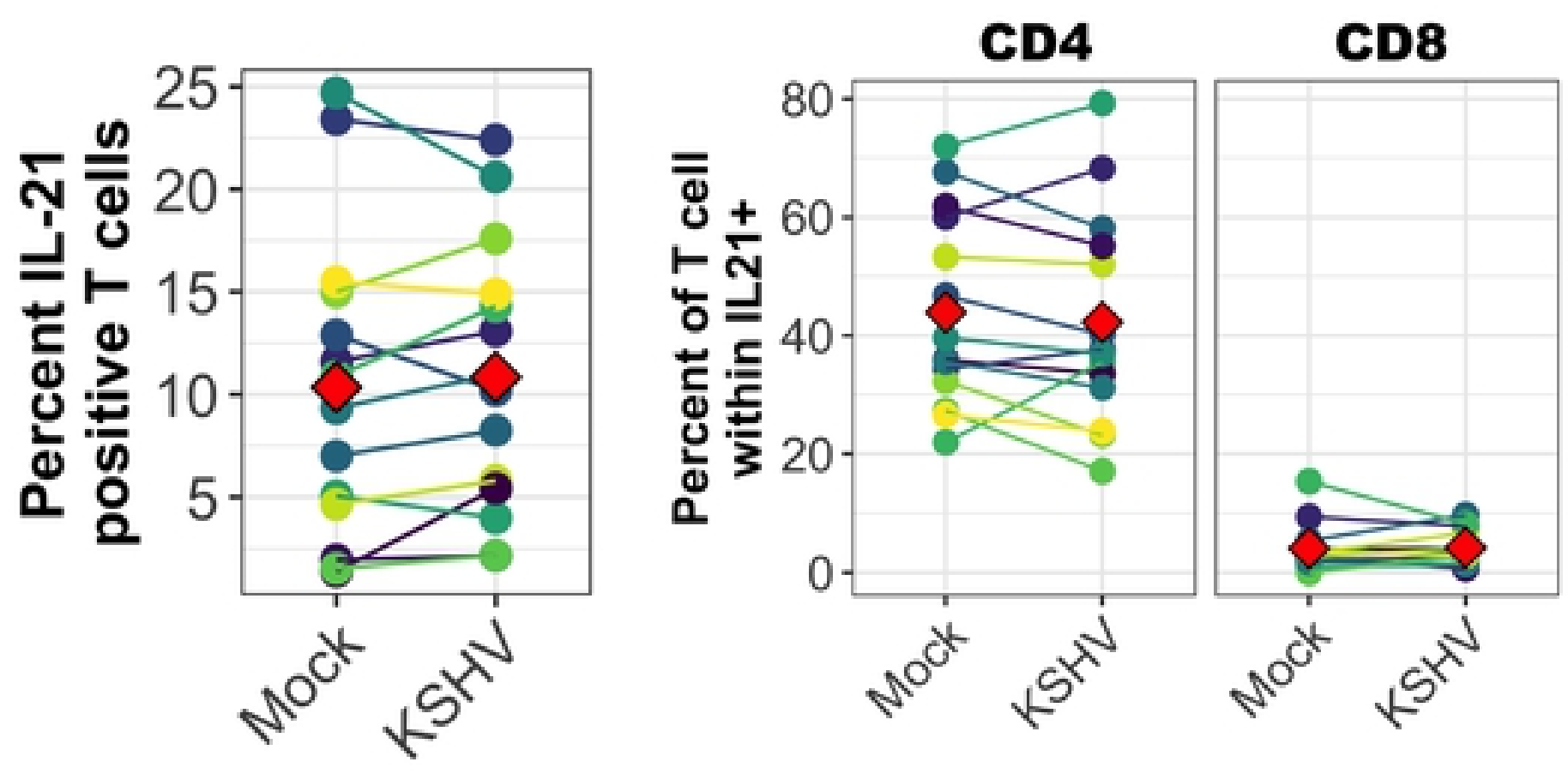

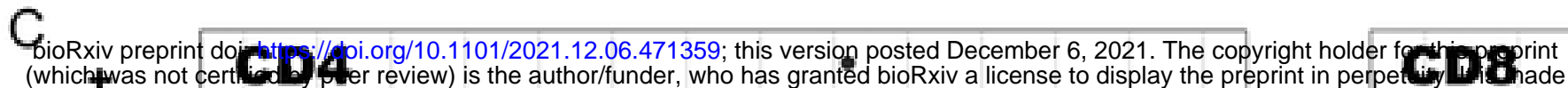

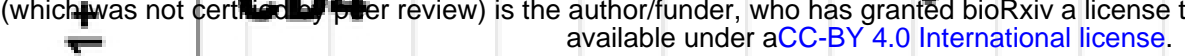
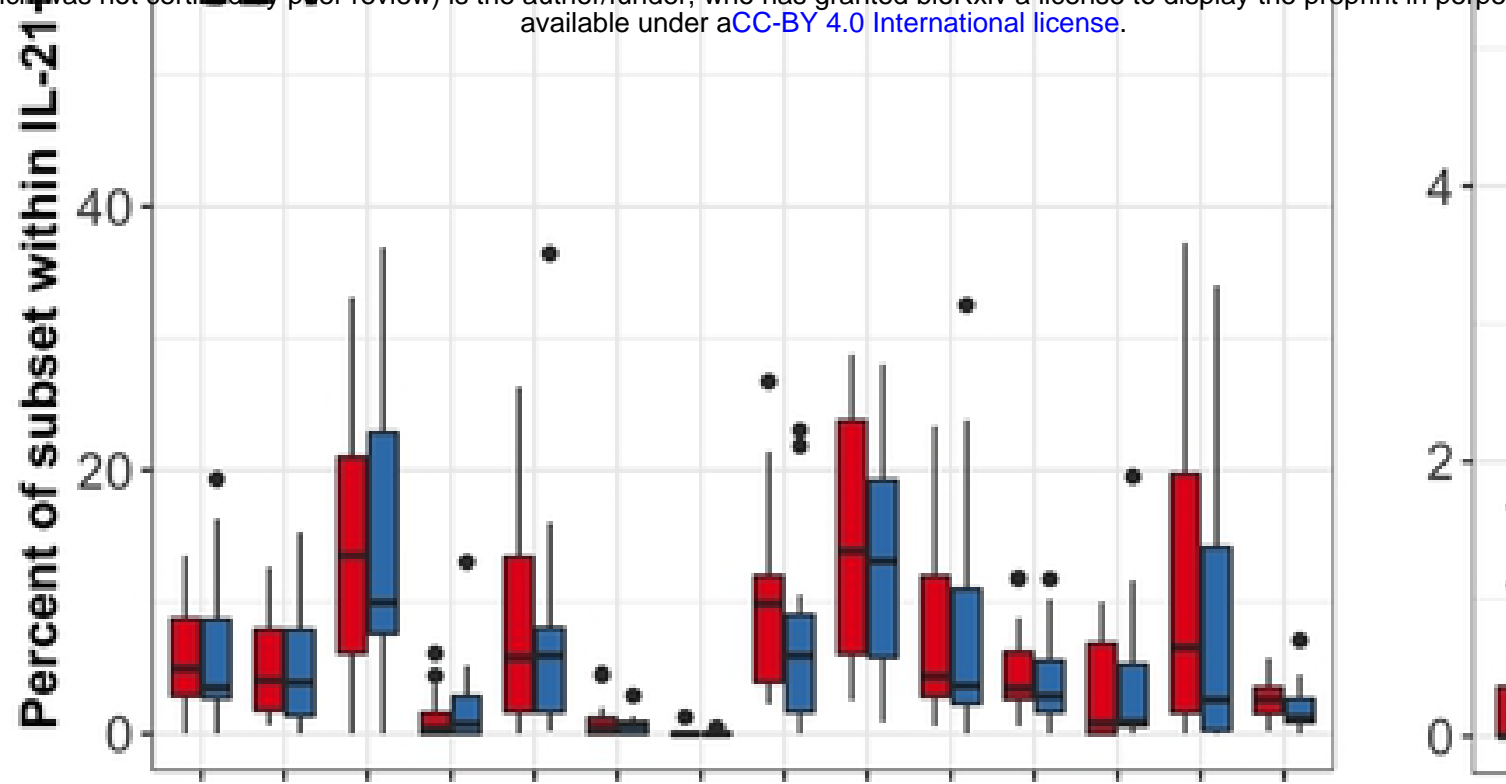

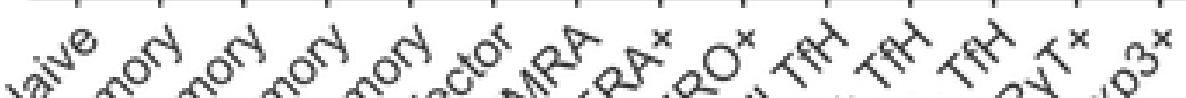

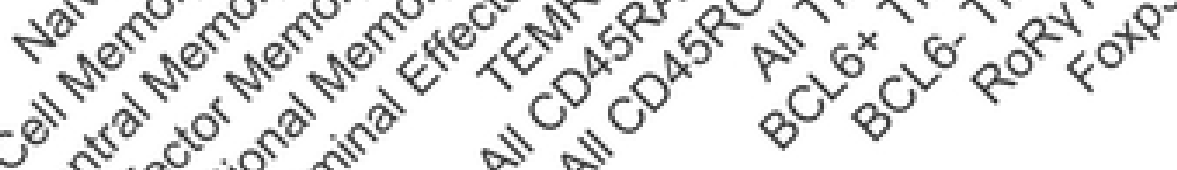
$2^{0}$
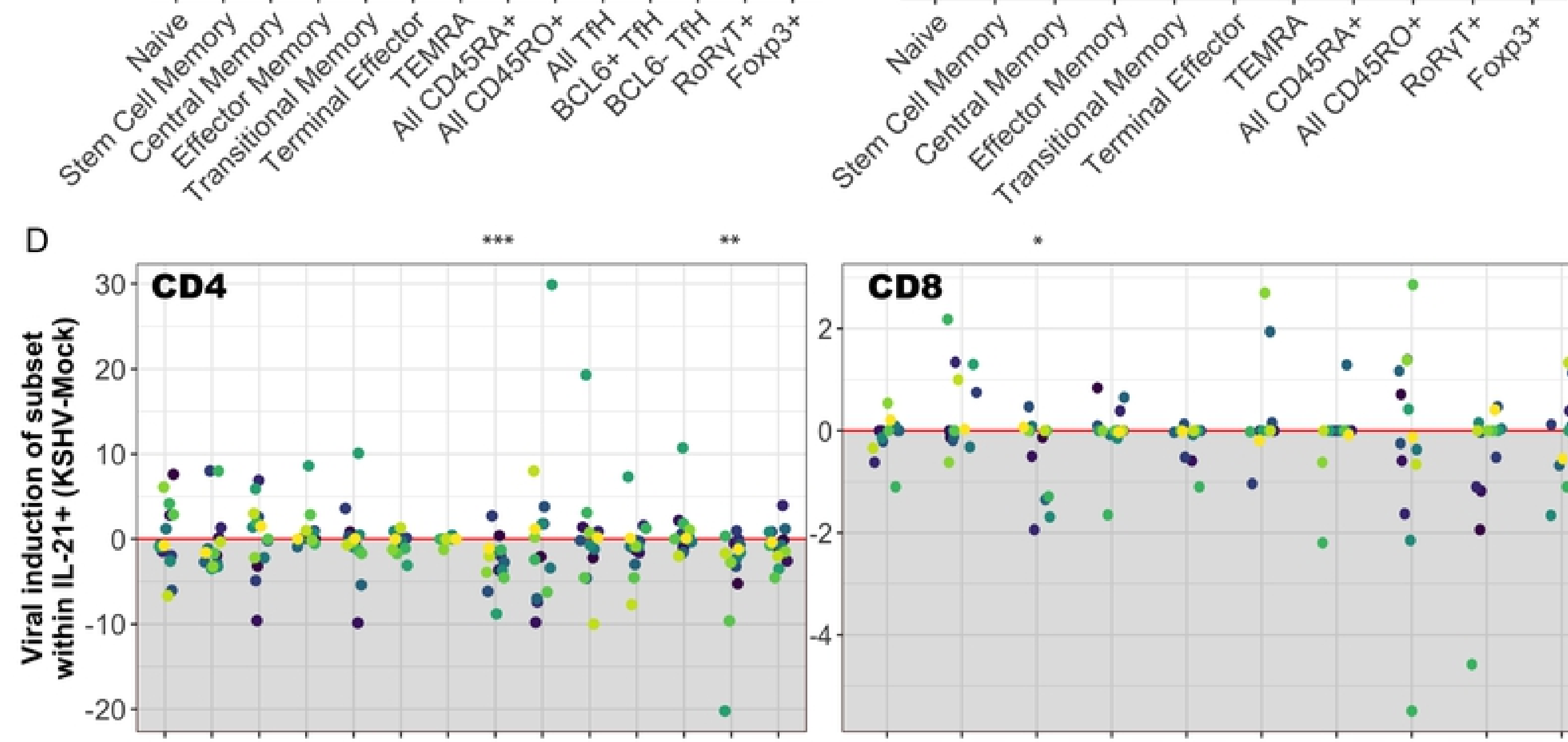

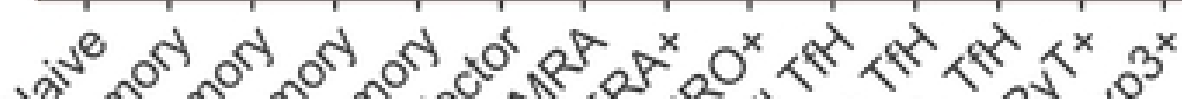
b. 20
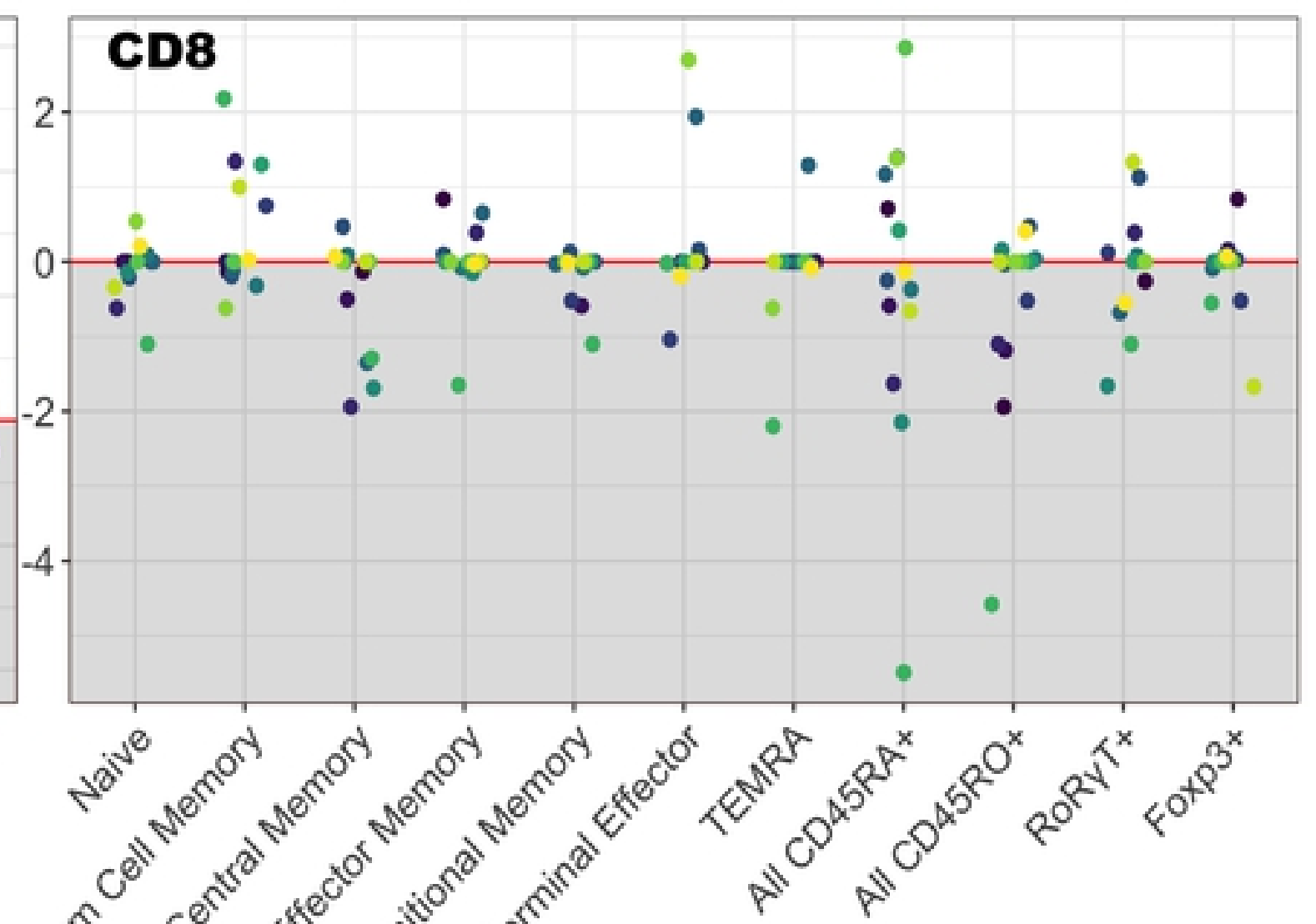

Figure 5 
